\title{
Demazure crystals, Kirillov-Reshetikhin crystals, and the energy function
}

\author{
Anne Schilling* \\ Department of Mathematics \\ University of California \\ One Shields Ave \\ Davis, CA 95616-8633, U.S.A. \\ anne@math.ucdavis.edu
}

\author{
Peter Tingley ${ }^{\dagger}$ \\ Department of Mathematics \\ M.I.T \\ Cambridge, MA 02139-4307, U.S.A. \\ peter.tingley@gmail.com
}

Submitted: Oct 13, 2011; Accepted: Mar 28, 2012; Published: Apr 7, 2012

Mathematics Subject Classifications: Primary 81R50, 81R10; Secondary: 05E99

\begin{abstract}
It has previously been shown that, at least for non-exceptional Kac-Moody Lie algebras, there is a close connection between Demazure crystals and tensor products of Kirillov-Reshetikhin crystals. In particular, certain Demazure crystals are isomorphic as classical crystals to tensor products of Kirillov-Reshetikhin crystals via a canonically chosen isomorphism. Here we show that this isomorphism intertwines the natural affine grading on Demazure crystals with a combinatorially defined energy function. As a consequence, we obtain a formula of the Demazure character in terms of the energy function, which has applications to Macdonald polynomials and $q$-deformed Whittaker functions.
\end{abstract}

\section{Contents}

1 Introduction $\quad 3$

1.1 Results . . . . . . . . . . . . . . . . . . . . 4

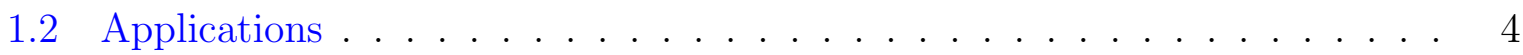

1.3 Exceptional types . . . . . . . . . . . . . . . . . . 5

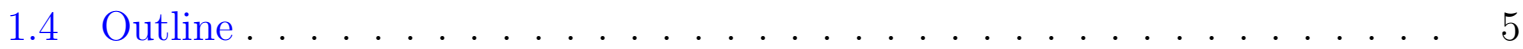

*Supported in part by NSF grants DMS-0652641, DMS-0652652, and DMS-1001256.

${ }^{\dagger}$ Supported in part by NSF grant DMS-0902649. 
2 Kac-Moody algebras and Crystals 5

2.1 General setup . . . . . . . . . . . . . . . . . . 5

$2.2 U_{q}(\mathfrak{g})$ crystals . . . . . . . . . . . . . . . . . . 6

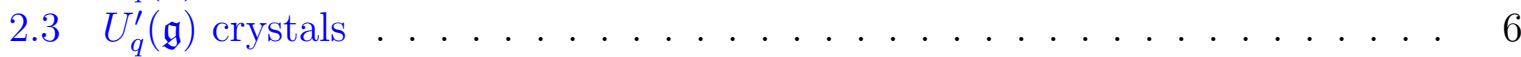

2.4 Tensor products of crystals . . . . . . . . . . . . . . . . . . . 7

2.5 Abstract crystals . . . . . . . . . . . . . . . . . . 7

2.6 Perfect crystals . . . . . . . . . . . . . . . . . . . . . 7

2.7 Kirillov-Reshetikhin modules and their crystals . . . . . . . . . . . . 9

2.8 Extended affine Weyl group . . . . . . . . . . . . . . . . . . . 10

2.9 Demazure modules and crystals . . . . . . . . . . . . . . . . . 11

3 Kashiwara-Nakashima tableaux and branching rules 13

3.1 Kashiwara-Nakashima tableaux . . . . . . . . . . . . . . . . . . . . 13

3.2 Branching rules and \pm diagrams . . . . . . . . . . . . . . . . . . . . . 14

3.3 Nested \pm diagrams $\ldots \ldots \ldots \ldots \ldots \ldots$

4 Realizations of KR crystals $\quad 18$

4.1 Classical decompositions of KR crystals . . . . . . . . . . . . . . 18

4.2 Classically irreducible KR crystals (case (IRR)) . . . . . . . . . . . . 19

4.3 KR crystals constructed via Dynkin automorphisms (case (AUT)) . . . . 19

$4.4 B^{r, s}$ of type $C_{n}^{(1)}$ for $r<n$ (in case (VIR) $\ldots \ldots \ldots \ldots$

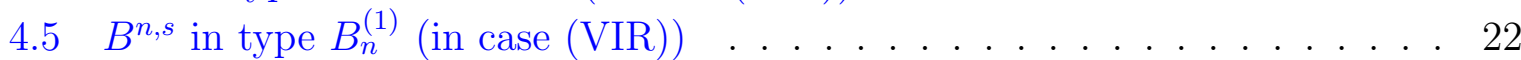

4.6 The KR crystals of type $A_{2 n}^{(2)}$ and $D_{n+1}^{(2)}$ in case (VIR) $\ldots \ldots \ldots 23$

5 Energy functions 24

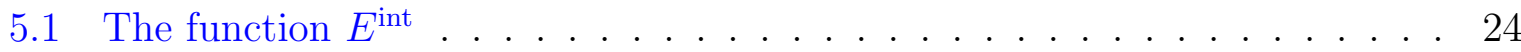

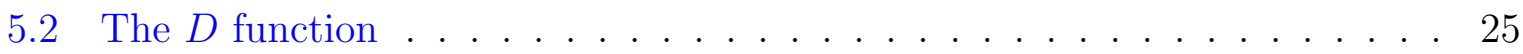

6 Perfect KR crystals and Demazure crystals 26

$\begin{array}{lll}7 & \text { The affine grading via the energy function } & 30\end{array}$

8 Generalizations to other tensor products of KR crystals 32

9 Applications 33

9.1 Demazure characters . . . . . . . . . . . . . . . . 34

9.2 Nonsymmetric Macdonald polynomials . . . . . . . . . . . . . . . . 35

$9.3 q$-deformed Whittaker functions . . . . . . . . . . . . . . 38 


\section{Introduction}

Kashiwara's theory of crystal bases [28] provides a remarkable combinatorial tool for studying highest weight representations of symmetrizable Kac-Moody algebras and their quantizations. Here we consider finite-dimensional representations of the quantized universal enveloping algebra $U_{q}^{\prime}(\mathfrak{g})$ corresponding to the derived algebra $\mathfrak{g}^{\prime}$ of an affine KacMoody algebra. These representations do not extend to representations of $U_{q}(\mathfrak{g})$, but one can nonetheless define the notion of a crystal basis. In this setting crystal bases do not always exist, but there is an important class of finite-dimensional modules for $U_{q}^{\prime}(\mathfrak{g})$ that are known to admit crystal bases: tensor products of the Kirillov-Reshetikhin modules $W^{r, s}$ from [34] (denoted $W\left(s \omega_{r}\right)$ in that paper), where $r$ is a node in the classical Dynkin diagram and $s$ is a positive integer.

The modules $W^{r, s}$ were first conjectured to admit crystal bases $B^{r, s}$ in $[17$, Conjecture 2.1], and moreover it was conjectured that these crystals are perfect whenever $s$ is a multiple of a particular constant $c_{r}$ (perfectness is a technical condition which allows one to use the finite crystal to construct highest weight crystals, see [25]). This conjecture has now been proven in all non-exceptional cases (see $[49,50]$ for a proof that the crystals exist, and [11, Theorem 1.2] for a proof that they are perfect). We call $B^{r, s}$ a KirillovReshetikhin (KR) crystal.

The perfectness of KR crystals ensures that they are related to highest weight affine crystals via the construction in [25]. In [31], Kashiwara proposed that this relationship is connected to the theory of Demazure crystals [27, 42], by conjecturing that perfect KR crystals are isomorphic as classical crystals to certain Demazure crystals (which are subcrystals of affine highest weight crystals). This was proven in most cases in [8, 9]. More relations between Demazure crystals and tensor products of perfect KR crystals were investigated in [36, 37, 38, 12, 47].

There is a natural grading deg on a highest weight affine crystal $B(\Lambda)$, where $\operatorname{deg}(b)$ records the number of $f_{0}$ in a string of $f_{i}$ 's that act on the highest weight element to give $b$ (this is well-defined by weight considerations). Due to the ideas discussed above, it seems natural that this grading should transfer to a grading on a tensor product of KR crystals.

Gradings on tensor products of KR crystals have in fact been studied, and are usually referred to as "energy functions." They are vast generalizations of the major index

statistics on words, which can be viewed as elements in $\left(B^{1,1}\right)^{\otimes L}$. The idea dates to the earliest works on perfect crystals [25, 26], and was expanded in [51] following conjectural definitions in [16]. A function $D$, which we will refer to as the $D$-function, is defined as a sum involving local energy functions for each pair of factors in the tensor product and an 'intrinsic energy' of each factor. It has been suggested that there is a simple global characterization of intrinsic energy related to the affine grading on a corresponding highest weight crystal (see [52, Section 2.5], [16, Proof of Proposition 3.9]). However, as far as we know, it has previously never been shown that the explicit definition of intrinsic energy actually satisfies this condition. 


\section{$1.1 \quad$ Results}

In the present work, we restrict to non-exceptional type (i.e. all affine Kac-Moody algebras except $A_{2}^{(2)}, G_{2}^{(1)}, F_{4}^{(1)}, E_{6}^{(1)}, E_{7}^{(1)}, E_{8}^{(1)}, E_{6}^{(2)}$ and $D_{4}^{(3)}$ ), where KR crystals are known to exist. The relationship between KR crystals and Demazure crystals discussed above can be enhanced: There is in fact a unique isomorphism of classical crystals between any tensor product $B=B_{1} \otimes \cdots \otimes B_{k}$ of perfect level $\ell$ KR crystals and a certain Demazure crystal such that all 0 arrows on the Demazure side correspond to 0 arrows on the KR sides (although there are more 0 arrows in the KR crystal). See Theorem 6.1 for the precise statement. This was proven by Fourier, Shimozono and the first author in [12, Theorem 4.4] under certain assumptions since at the time KR crystals were not yet known to exist. Here we point out that in most cases the assumptions from [12] follow from $[49,50,10,11]$. In the remaining cases of type $A_{2 n}^{(2)}$ and the spin nodes for type $D_{n}^{(1)}$ we prove the statement separately, thereby firmly establishing this relationship between KR crystals and Demazure crystals in all non-exceptional types.

We then establish the correspondence between the $D$ function and the affine grading discussed earlier in this introduction. That is, we show that the unique isomorphism discussed above intertwines the basic grading on the Demazure crystal with the $D$-function on the KR crystal, up to a shift (i.e. addition of a global constant). This also allows for a new characterization of the $D$ function. Define the intrinsic energy function $E^{\text {int }}$ on $B$ by letting $E^{\text {int }}(b)$ record the minimal number of $f_{0}$ in a path from a certain fixed $u \in B$ to $b$. We show that $E^{\text {int }}$ agrees with the $D$-function up to a shift (i.e. addition of a global constant). The isomorphism between KR crystals and Demazure crystals actually intertwines the basic grading with $E^{\text {int }}$ exactly. In particular this shows that for any Demazure crystal arrow (see Remark 7.7) the energy changes by 1 on the corresponding classical crystal components.

We also consider the more general setting when $B$ is a tensor product of KR crystals which are not assumed to be perfect or of the same level. The $D$ function is still welldefined, and we give a precise relationship between $D$ and the affine grading on a related direct sum of highest weight modules in Corollaries 8.3 and 8.4. In this case we no longer give an interpretation in terms of Demazure modules, although for tensor products of perfect crystals of various levels it was subsequently shown by Naoi [47] that the result is the disjoint union of Demazure crystals; see also Remark 8.5.

\subsection{Applications}

Our results express the characters of certain Demazure modules in terms of the intrinsic energy on a related tensor product of KR crystals (see Corollary 9.1). This has potential applications whenever those Demazure characters appear.

For untwisted simply-laced root systems, Ion [21], generalizing results of Sanderson [53] in type $A$, showed that the specializations $E_{\lambda}(q, 0)$ of nonsymmetric Macdonald polynomials at $t=0$ coincide with specializations of Demazure characters of level one affine integrable modules. If $\lambda$ is anti-dominant, then $E_{\lambda}(q, 0)$ is actually a symmetric Macdon- 
ald polynomial $P_{\lambda}(q, 0)$. In this case, the relevant Demazure module is associated to a tensor product $B$ of level one KR crystals as above, so our results imply that $P_{\lambda}(q, 0)$ is the character of $B$, where the powers of $q$ are given by $-D$, see Corollary 9.5. It follows that the coefficients in the expansion of $P_{\lambda}(q, 0)$ in terms of the irreducible characters are the one-dimensional configuration sums defined in terms of the intrinsic energy in [16].

There is also a relation between Demazure characters and $q$-deformed Whittaker functions for $\mathfrak{g l}_{n}[13$, Theorem 3.2]. Hence our results allow one to study Whittaker functions via $\mathrm{KR}$ crystals.

\section{$1.3 \quad$ Exceptional types}

KR crystals are still expected to exist in exceptional types, although in most cases this has not yet been established (see for example [22, 30, 32, 56] for some cases where it has). Furthermore, it is expected that the relationship between KR crystals and Demazure crystals holds in general (see [31] for the conjecture, and [8, 9] for a proof that it holds in some exceptional cases). Our expectation is that Theorem 6.1 and Corollary 7.6 would also continue to hold in all cases.

\subsection{Outline}

In Section 2 we briefly review the theory of crystals. In Section 3 we review combinatorial models for non-exceptional finite type crystals and their branching rules. Section 4 is devoted to combinatorial models for KR crystals. In Section 5, we introduce the two energy functions $E^{\text {int }}$ and $D$. Section 6 discusses the isomorphism between Demazure crystals and tensor products of perfect KR crystals. In Section 7 we relate the basic grading on a Demazure crystal with the energy function on a tensor product of KR crystals and show that the two energy functions agree up to a shift (Theorems 7.4 and 7.5). Some of these results are generalized to tensor products of (not necessarily perfect) KR crystals of different level in Section 8. In Section 9 we discuss applications to Demazure characters, nonsymmetric Macdonald polynomials, and Whittaker functions.

\section{Kac-Moody algebras and Crystals}

\subsection{General setup}

Let $\mathfrak{g}$ be a Kac-Moody algebra. Let $\Gamma=(I, E)$ be its Dynkin diagram, where $I$ is the set of vertices and $E$ the set of edges. Let $\Delta$ be the corresponding root system and $\left\{\alpha_{i} \mid i \in I\right\}$ the set of simple roots. Let $P, Q, P^{\vee}$, and $Q^{\vee}$ denote the weight lattice, root lattice, coweight lattice, and coroot lattice respectively.

Let $U_{q}(\mathfrak{g})$ be the corresponding quantum enveloping algebra over $\mathbb{Q}(q)$. Let $\left\{E_{i}, F_{i}\right\}_{i \in I}$ be the standard elements in $U_{q}(\mathfrak{g})$ corresponding to the Chevalley generators of the derived algebra $\mathfrak{g}^{\prime}$. We recall the triangular decomposition

$$
U_{q}(\mathfrak{g}) \cong U_{q}(\mathfrak{g})^{<0} \otimes U_{q}(\mathfrak{g})^{0} \otimes U_{q}(\mathfrak{g})^{>0},
$$


where $U_{q}(\mathfrak{g})^{<0}$ is the subalgebra generated by the $F_{i}, U_{q}(\mathfrak{g})^{>0}$ is the subalgebra generated by the $E_{i}, U_{q}(\mathfrak{g})^{0}$ is the abelian group algebra generated by the usual elements $K_{w}$ for $w \in P^{\vee}$, and the isomorphism is as vector spaces. Let $U_{q}^{\prime}(\mathfrak{g})$ be the subalgebra generated by $E_{i}, F_{i}$ and $K_{i}:=K_{\alpha_{i}^{\vee}}$ for $i \in I$.

We are particularly interested in the case when $\mathfrak{g}$ is of affine type. In that situation, we use the following conventions: $\Lambda_{i}$ to denotes the fundamental weight corresponding to $i \in I$. For $i \in I \backslash\{0\}, \omega_{i}$ denotes the fundamental weight corresponding to that node in the related finite type Lie algebra. The corresponding finite type weight lattice and Weyl group are denoted $\bar{P}$ and $\bar{W}$, respectively.

\section{$2.2 \quad U_{q}(\mathfrak{g})$ crystals}

Here we give a very quick review, and refer the reader to [18] more details. For us, a crystal is a nonempty set $B$ along with operators $e_{i}: B \rightarrow B \cup\{0\}$ and $f_{i}: B \rightarrow B \cup\{0\}$ for $i \in I$, which satisfy some conditions. The set $B$ records certain combinatorial data associated to a representation $V$ of $U_{q}(\mathfrak{g})$, and the operators $e_{i}$ and $f_{i}$ correspond to the Chevalley generators $E_{i}$ and $F_{i}$. The relationship between the crystal $B$ and the module $V$ can be made precise using the notion of a crystal basis for $V$.

Often the definition of a crystal includes three functions wt, $\varphi, \varepsilon: B \rightarrow P$, where $P$ is the weight lattice. In the case of crystals of integrable modules, these functions can be recovered (up to a global shift in a null direction in cases where the Cartan matrix is not invertible) from the knowledge of $e_{i}$ and $f_{i}$, as we discuss in a slightly different context in Section 2.3.

An important theorem of Kashiwara shows that every integrable $U_{q}(\mathfrak{g})$ highest weight module $V(\lambda)$ has a crystal basis and hence a corresponding crystal $B(\lambda)$.

\section{$2.3 \quad U_{q}^{\prime}(\mathfrak{g})$ crystals}

In the case when the Cartan matrix is not invertible, one can define an extended notion of $U_{q}^{\prime}(\mathfrak{g})$ crystal bases and crystals that includes some cases which do not lift to $U_{q}(\mathfrak{g})$ crystals. Note however that not all integrable $U_{q}^{\prime}(\mathfrak{g})$ representations have corresponding crystals. See e.g. [30]. We consider only integrable crystals $B$, i.e. crystals where each $e_{i}, f_{i}$ acts locally nilpotently. Define a weight function on such a $B$ as follows: First set

$$
\begin{aligned}
\varepsilon_{i}(b) & :=\max \left\{m \mid e_{i}^{m}(b) \neq 0\right\}, \\
\varphi_{i}(b) & :=\max \left\{m \mid f_{i}^{m}(b) \neq 0\right\} .
\end{aligned}
$$

Let $\Lambda_{i}$ be the fundamental weight associated to $i \in I$. For each $b \in B$, define

$$
\begin{aligned}
\text { (i) } \varphi(b) & :=\sum_{i \in I} \varphi_{i}(b) \Lambda_{i}, \\
\text { (ii) } \varepsilon(b) & :=\sum_{i \in I} \varepsilon_{i}(b) \Lambda_{i},
\end{aligned}
$$


(iii) $\operatorname{wt}(b):=\varphi(b)-\varepsilon(b)$.

Then wt $(b)$ corresponds to the classical weight grading of the $U_{q}(\mathfrak{g})$ module associated to $B$, and is referred to as the weight function. Notice that wt $(b)$ is always in

$$
P^{\prime}:=\operatorname{span}\left\{\Lambda_{i}: i \in I\right\}
$$

If the Cartan matrix of $\mathfrak{g}$ is not invertible, $P^{\prime}$ is a proper sublattice of $P$.

Remark 2.1. One would expect that $f_{i}$ should have weight $-\alpha_{i}$. This is true for $U_{q}(\mathfrak{g})$ crystals, but for $U_{q}^{\prime}(\mathfrak{g})$ crystals the weight of $f_{i}$ is actually the projection of $-\alpha_{i}$ onto $P^{\prime}$ under the projection that sends null roots to 0 .

\subsection{Tensor products of crystals}

The tensor product rule for $U_{q}^{\prime}(\mathfrak{g})$ or $U_{q}(\mathfrak{g})$ modules leads to a tensor product rule for the corresponding crystals. Following [12], we use the opposite conventions from Kashiwara [28]. If $A$ and $B$ are two crystals, the tensor product $A \otimes B$ is the crystal whose underlying set is the Cartesian set $\{a \otimes b \mid a \in A, b \in B\}$ with operators $e_{i}$ and $f_{i}$ defined by:

$$
\begin{aligned}
& e_{i}(a \otimes b)= \begin{cases}e_{i}(a) \otimes b & \text { if } \varepsilon_{i}(a)>\varphi_{i}(b), \\
a \otimes e_{i}(b) & \text { otherwise, }\end{cases} \\
& f_{i}(a \otimes b)= \begin{cases}f_{i}(a) \otimes b & \text { if } \varepsilon_{i}(a) \geqslant \varphi_{i}(b), \\
a \otimes f_{i}(b) & \text { otherwise. }\end{cases}
\end{aligned}
$$

\subsection{Abstract crystals}

Definition 2.2. Let $\mathfrak{g}$ be a Kac-Moody algebra with Dynkin diagram $\Gamma=(I, E)$. Let $B$ be a nonempty set with operators $e_{i}, f_{i}: B \rightarrow B \sqcup\{0\}$. We say $B$ is a regular abstract crystal of type $\mathfrak{g}$ if, for each pair $i \neq j \in I, B$ along with the operators $e_{i}, f_{i}, e_{j}, f_{j}$ is a union of (possibly infinitely many) integrable highest weight $U_{q}\left(\mathfrak{g}_{i, j}\right)$ crystals, where $\mathfrak{g}_{i, j}$ is the Lie algebra with Dynkin diagram containing $i$ and $j$, and all edges between them.

Remark 2.3. If $\mathfrak{g}$ is an affine algebra other than $\widehat{\mathfrak{s l}}_{2}$, then any $U_{q}^{\prime}(\mathfrak{g})$ crystal is a regular abstract crystal of type $\mathfrak{g}$.

\subsection{Perfect crystals}

In this section, $\mathfrak{g}$ is of affine type. Let $c=\sum_{i \in I} a_{i}^{\vee} \alpha_{i}^{\vee}$ be the canonical central element associated to $\mathfrak{g}$ and $P^{+}$the set of dominant weights, that is, $P^{+}=\left\{\Lambda \in P \mid \Lambda\left(\alpha_{i}^{\vee}\right) \in \mathbb{Z}_{\geqslant 0}\right\}$. We define the level of $\Lambda \in P^{+}$by $\operatorname{lev}(\Lambda):=\Lambda(c)$. For each $\ell \in \mathbb{Z}$, we consider the sets

$$
P_{\ell}=\{\Lambda \in P \mid \operatorname{lev}(\Lambda)=\ell\} \quad \text { and } \quad P_{\ell}^{+}=\left\{\Lambda \in P^{+} \mid \operatorname{lev}(\Lambda)=\ell\right\},
$$

the sets of level- $\ell$ weights and level- $\ell$ dominant weights respectively. Note that $P_{\ell}^{+}=\emptyset$ if $\ell<0$. The following important notion was introduced in [25]. 


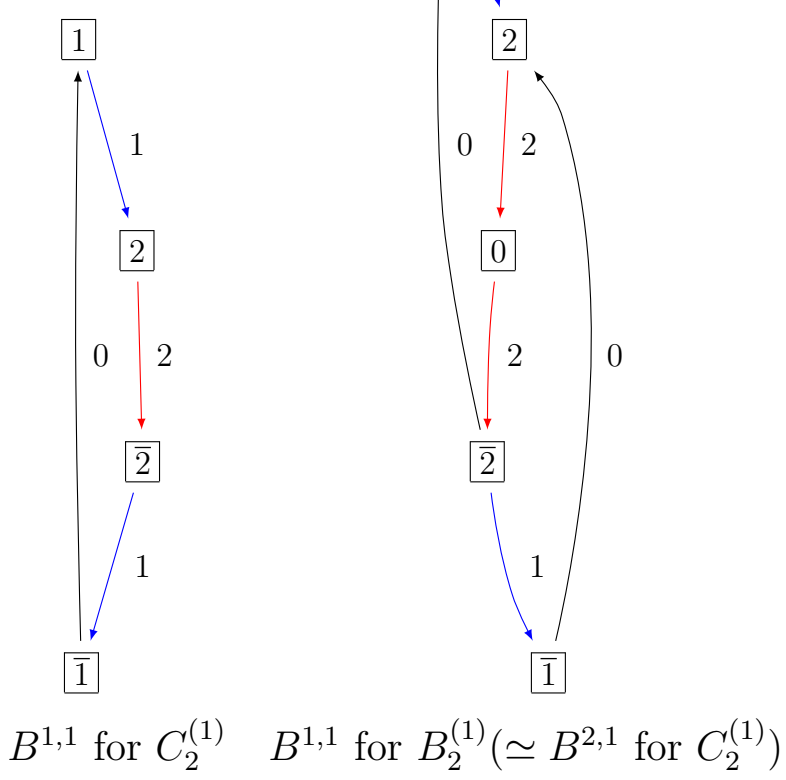

Figure 1: Examples of perfect and non-perfect $U_{q}^{\prime}(\mathfrak{g})$ crystals. One can verify that the crystal on the right is perfect of level 1, and the crystal on the left is not perfect of any level. These crystals are both KR crystals, as indicated, and the vertices are indexed by $\mathrm{KN}$ tableaux as in Section 3.1. In fact, all known finite $U_{q}^{\prime}(\mathfrak{g})$ crystals are KR crystals or tensor products of KR crystals.

Definition 2.4. For a positive integer $\ell>0$, a $U_{q}^{\prime}(\mathfrak{g})$-crystal $B$ is called a perfect crystal of level $\ell$ if the following conditions are satisfied:

(i) $B$ is isomorphic to the crystal graph of a finite-dimensional $U_{q}^{\prime}(\mathfrak{g})$-module.

(ii) $B \otimes B$ is connected.

(iii) There exists a $\lambda \in \bar{P}$, such that wt $(B) \subset \lambda+\sum_{i \in I \backslash\{0\}} \mathbb{Z}_{\leqslant 0} \alpha_{i}$ and there is a unique element in $B$ of classical weight $\lambda$.

(iv) $\forall b \in B, \operatorname{lev}(\varepsilon(b)) \geqslant \ell$.

(v) $\forall \Lambda \in P_{\ell}^{+}$, there exist unique elements $b_{\Lambda}, b^{\Lambda} \in B$, such that

$$
\varepsilon\left(b_{\Lambda}\right)=\Lambda=\varphi\left(b^{\Lambda}\right) .
$$

Examples of a perfect and nonperfect crystal is given in Figure 1. 


\subsection{Kirillov-Reshetikhin modules and their crystals}

In this section $\mathfrak{g}$ is of affine type. The Kirillov-Reshetikhin modules were first introduced for the Yangian of $\mathfrak{g}^{\prime}$ in [34]. One can characterize the KR module $W^{r, s}$ for $U_{q}^{\prime}(\mathfrak{g})[5,6]$, where $r \in I \backslash\{0\}$ and $s \geqslant 1$, as the irreducible representations of $U_{q}^{\prime}(\mathfrak{g})$ whose Drinfeld polynomials are given by

$$
P_{i}(u)= \begin{cases}\left(1-q_{i}^{1-s} u\right)\left(1-q_{i}^{3-s} u\right) \cdots\left(1-q_{i}^{s-1} u\right) & \text { if } i=r, \\ 1 & \text { otherwise. }\end{cases}
$$

Here $q_{i}=q^{\left(\alpha_{i} \mid \alpha_{i}\right) / 2}$.

It was shown in [26] for type $A_{n}^{(1)}$ and many special cases, and in $[49,50]$ for general non-exceptional types, that the modules $W^{r, s}$ have crystal bases. We denote the resulting crystals by $B^{r, s}$, and refer to them as $K R$ crystals.

Theorem 2.5. [50, 11] In all non-exceptional types, $W^{r, s}$ has a crystal base $B^{r, s}$. Furthermore, if $s$ is a multiple of $c_{r}$ (see Figure 4) the resulting crystals are perfect.

The following is a slight strengthening of results from [10].

Lemma 2.6. Let $\mathfrak{g}$ be a non-exceptional affine Kac-Moody algebra with index set $I=$ $\{0,1, \ldots, n\}$. Fix $r \in I \backslash\{0\}$ and $s>0$. Then any regular abstract crystal $B$ (see Definition 2.2) of type $\mathfrak{g}$ which is isomorphic to $B^{r, s}$ as a $\{1,2, \ldots, n\}$-crystal is also isomorphic to $B^{r, s}$ as an I-crystal.

Proof. First, recall that any finite type crystal $C$ is uniquely determined by its character

$$
\operatorname{ch}(C):=\sum_{c \in C} e^{\mathrm{wt}}{ }^{\mathrm{fin}(c)},
$$

where wt ${ }^{\text {fin }}$ denotes the weight as a $\{1,2, \ldots, n\}$-crystal. Since $B$ is isomorphic to $B^{r, s}$ as a $\{1,2, \ldots, n\}$-crystal, it is finite and hence of level 0 . Thus wt $(b)$ can be recovered from $\mathrm{wt}^{\text {fin }}(b)$. In particular, we can recover the character of $B$ as an $I \backslash\{j\}$ crystal for all $j \in I$, and from there recover the isomorphism class of $B$ as an $I \backslash\{j\}$-crystal.

The lemma now follows by the following uniqueness results from $[50,10]$ :

- By [50, Proposition 6.1] (for most nodes) and [10] (for exceptional nodes), in types $D_{n}^{(1)}, B_{n}^{(1)}$ and $A_{2 n-1}^{(2)}$, any regular abstract crystal $B$ which is isomorphic to $B^{r, s}$ as both a $\{1,2, \ldots, n\}$-crystal and a $\{0,2, \ldots, n\}$-crystal is isomorphic to $B^{r, s}$ as an affine crystal.

- As in [10, Sections 5.2 and 6.1], in types $C_{n}^{(1)}, D_{n+1}^{(2)}$ and $A_{2 n}^{(2)}$, any regular abstract crystal $B$ which is isomorphic to $B^{r, s}$ as both a $\{1,2, \ldots, n\}$-crystal and a $\{0,1, \ldots, n-1\}$-crystal is isomorphic to $B^{r, s}$ as an affine crystal. 
By [39, Proposition 3.8], a tensor product $B=B^{r_{1}, s_{1}} \otimes \cdots \otimes B^{r_{N}, s_{N}}$ of KR crystals is connected. We refer to such a $B$ as a composite $K R$ crystal. As in [25], if the factors are all perfect KR crystals of the same level, then $B=B^{r_{1}, \ell c_{r_{1}}} \otimes \cdots \otimes B^{r_{N}, \ell c_{r_{N}}}$ is also perfect of level $\ell$. We refer to such a perfect crystal as a composite KR crystal of level $\ell$.

Explicit combinatorial models for KR crystals of non-exceptional type were constructed in [10], and will be reviewed in Section 4. Two examples are given in Figure 1.

\subsection{Extended affine Weyl group}

Write the null root as $\delta=\sum_{i \in I} a_{i} \alpha_{i}$. Let $\theta=\delta-a_{0} \alpha_{0}$. As in [23, Section 6.4], $\theta$ is a root in the finite type root system corresponding to $I \backslash\{0\}$. Following [16], for each $i \in I \backslash\{0\}$, define $c_{i}=\max \left(1, a_{i} / a_{i}^{\vee}\right)$. It turns out that $c_{i}=1$ except for $c_{i}=2$ for: $\mathfrak{g}=B_{n}^{(1)}$ and $i=n, \mathfrak{g}=C_{n}^{(1)}$ and $1 \leqslant i \leqslant n-1, \mathfrak{g}=F_{4}^{(1)}$ and $i=3,4$, and $c_{2}=3$ for $\mathfrak{g}=G_{2}^{(1)}$. Here we use Kac's indexing of affine Dynkin diagrams from [23, Table Fin, Aff1 and Aff2]. Consider the sublattices of $\bar{P}$ given by

$$
\begin{aligned}
& M=\bigoplus_{i \in I \backslash\{0\}} \mathbb{Z} c_{i} \alpha_{i}=\mathbb{Z} \bar{W} \cdot \theta / a_{0}, \\
& \widetilde{M}=\bigoplus_{i \in I \backslash\{0\}} \mathbb{Z} c_{i} \omega_{i} .
\end{aligned}
$$

Let $\bar{W}$ be the finite type Weyl group for the Dynkin diagram $I \backslash\{0\}$, which acts on $\bar{P}$ by linearizing the rules $s_{i} \lambda=\lambda-\left\langle\alpha_{i}^{\vee}, \lambda\right\rangle \alpha_{i}$. Clearly $M \subset \widetilde{M}$ and the action of $\bar{W}$ on $\bar{P}$ restricts to actions on $M$ and $\widetilde{M}$. Let $T(\widetilde{M})$ (resp. $T(M)$ ) be the subgroup of $T(\bar{P})$ generated by the translations $t_{\lambda}$ by $\lambda \in \widetilde{M}$ (resp. $\lambda \in M$ ).

There is an isomorphism [23, Prop. 6.5]

$$
W \cong \bar{W} \ltimes T(M)
$$

as subgroups of $\operatorname{Aut}(\bar{P})$, where $W$ is the affine Weyl group. Under this isomorphism we have

$$
s_{0}=t_{\theta / a_{0}} s_{\theta},
$$

where $s_{\theta}$ is the reflection corresponding to the root $\theta$. Define $\theta^{\vee} \in \mathfrak{h}^{*}$ so that $s_{\theta}(\lambda)=$ $\lambda-\left\langle\theta^{\vee}, \lambda\right\rangle \theta$.

Define the extended affine Weyl group to be the subgroup of $\operatorname{Aut}(P)$ given by

$$
\widetilde{W}=\bar{W} \ltimes T(\widetilde{M}) .
$$

Let $C \subset \bar{P} \otimes_{\mathbb{Z}} \mathbb{R}$ be the fundamental chamber, the set of elements $\lambda$ such that $\left\langle\alpha_{i}^{\vee}, \lambda\right\rangle \geqslant 0$ for all $i \in I \backslash\{0\}$, and $\left\langle\theta^{\vee}, \lambda\right\rangle \leqslant 1 / a_{0}$.

Let $\Sigma \subset \widetilde{W}$ be the subgroup of $\widetilde{W}$ consisting of those elements that send $C$ into itself. Then $\widetilde{W}=W \Sigma$, and in particular every element $x \in \widetilde{W}$ can be written uniquely as

$$
x=w \tau
$$

THE ELECtronic Journal of COMBinatorics 19(2) (2012), \#P4 
for some $w \in W$ and $\tau \in \Sigma$.

The usual affine Weyl group $W$ is a normal subgroup of $\widetilde{W}$, so $\Sigma$ acts on $W$ by conjugation. Each $\tau \in \Sigma$ induces an automorphism (also denoted $\tau$ ) of the affine Dynkin diagram $\Gamma$, which is characterized as the unique automorphism so that:

$$
\tau s_{i} \tau^{-1}=s_{\tau(i)} \quad \text { for each } i \in I .
$$

Remark 2.7. When $\mathfrak{g}$ is of untwisted type, $M \cong Q^{\vee}, \widetilde{M} \cong P^{\vee}$, with the isomorphism $\nu$ given by $c_{i} \omega_{i}=\nu\left(\omega_{i}^{\vee}\right)$, and $c_{i} \alpha_{i}=\nu\left(\alpha_{i}^{\vee}\right)$ for $i \in I \backslash\{0\}$.

Lemma 2.8. Fix $\mathfrak{g}$ with affine Dynkin diagram $\Gamma$, and let $\tau \in A u t(\Gamma)$. Then $\tau \in \Sigma$ if and only if there exists $w_{\tau} \in \bar{W}$ such that the following diagram commutes

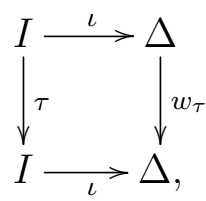

where $\Delta$ is the underlying finite type root system, and $\iota$ is the map that takes $i$ to $\alpha_{i}$ for all $i \neq 0$ and takes 0 to $-\theta$.

Proof. Fix $\tau \in \Sigma$. By (2.6), we can write $\tau=t_{\lambda} w$, where $w \in \bar{W}$, and $\lambda \in P^{\vee}$. Then $w$ must send $C$ to a chamber which can be shifted back to $C$, from which one can see that $w$ has the desired properties.

Now fix $\tau \in \operatorname{Aut}(\Gamma)$. If there is a $w_{\tau} \in \bar{W}$ making the diagram commute, then consider the element $t_{\omega_{\tau(0)}} w_{\tau} \in \widetilde{W}$. A simple calculation shows that $t_{\omega_{\tau(0)}} w_{\tau}(C)=C$, and that this realizes $\tau$ as an element of $\Sigma$. This last step uses the fact that $a_{\tau(0)}$ is always 1 when there is a non-trivial diagram automorphism $\tau$.

\subsection{Demazure modules and crystals}

In this section $\mathfrak{g}$ is an arbitrary symmetrizable Kac-Moody algebra. Let $\lambda$ be a dominant integral weight for $\mathfrak{g}$. Define

$$
W^{\lambda}:=\{w \in W \mid w \lambda=\lambda\} .
$$

Fix $\mu \in W \lambda$, and recall that the $\mu$ weight space in $V(\lambda)$ is one-dimensional. Let $u_{\mu}$ be a non-zero element of the $\mu$ weight space in $V(\lambda)$. Write $\mu=w \lambda$ where $w$ is the shortest element in the coset $w W^{\lambda}$. The Demazure module is defined to be

$$
V_{w}(\lambda):=U_{q}(\mathfrak{g})^{>0} \cdot u_{w(\lambda)},
$$

and the Demazure character is

$$
\operatorname{ch} V_{w}(\lambda)=\sum_{\mu} \operatorname{dim}\left(V_{w}(\lambda)_{\mu}\right) e^{\mu},
$$


where $V_{w}(\lambda)_{\mu}$ is the $\mu$ weight space of the Demazure module $V_{w}(\lambda)$.

It was conjectured by Littelmann [43] and proven by Kashiwara [27] that the intersection of a crystal basis of $V(\lambda)$ with $V_{w}(\lambda)$ is a crystal basis for $V_{w}(\lambda)$. The resulting subset $B_{w}(\lambda) \subset B(\lambda)$ is referred to as the Demazure crystal. It has the properties that it is closed under the action of the crystal operators $e_{i}$ (but not $f_{i}$ ), and that

$$
\operatorname{ch} V_{w}(\lambda)=\sum_{b \in B_{w}(\lambda)} e^{\mathrm{wt}(b)}
$$

Define the set

$$
f_{w}(b):=\left\{f_{i_{N}}^{m_{N}} \cdots f_{i_{1}}^{m_{1}}(b) \mid m_{k} \in \mathbb{Z}_{\geqslant 0}\right\}
$$

where $w=s_{i_{N}} \cdots s_{i_{1}}$ is any reduced decomposition of $w$. By [27, Proposition 3.2.3], as sets,

$$
B_{w}(\lambda)=f_{w}\left(u_{\lambda}\right)
$$

independent of the reduced word for $w$.

Example 2.9. Let us consider the Demazure crystal $B_{s_{2} s_{1}}\left(\omega_{1}+\omega_{2}\right)$ for $\mathfrak{s l}_{3}$. The Demazure crystal is shown with thick vertices and edges, will the rest of the ambient crystal $B\left(\omega_{1}+\right.$ $\left.\omega_{2}\right)$ is shown in thinner lines. Here blue lines show the action of $f_{1}$ and red lines show the action of $f_{2}$.

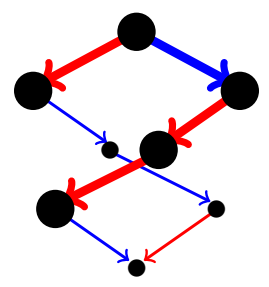

Remark 2.10. We mainly consider the case when $\mathfrak{g}$ is affine, and the following Demazure modules: Fix an anti-dominant weight $\mu \in \bar{P}$, and write $t_{\mu} \in \widetilde{W}$ as $t_{\mu}=w \tau$, where $w \in W$ and $\tau \in \Sigma$. For a dominant $\Lambda \in P$, we consider $V_{w}(\tau(\Lambda))$ and its crystal $B_{w}(\tau(\Lambda))$. In [12] these were denoted by $V_{\mu}(\Lambda)$ and $B_{\mu}(\Lambda)$, respectively.

Remark 2.11. It is well-known $[7,35,45]$ that the Demazure character can be expressed in terms of the Demazure operator $D_{i}: \mathbb{Z}[P] \rightarrow \mathbb{Z}[P]$

$$
D_{i}\left(e^{\mu}\right)=\frac{e^{\mu}-e^{\mu-\left(1+\left\langle\alpha_{i}^{\vee}, \mu\right\rangle\right) \alpha_{i}}}{1-e^{-\alpha_{i}}},
$$

where $\alpha_{i}$ is a simple root and $\alpha_{i}^{\vee}$ the corresponding coroot. Then for $w=s_{i_{N}} \cdots s_{i_{1}} \in W$ a reduced expression

$$
\operatorname{ch} V_{w}(\lambda)=D_{i_{N}} \cdots D_{i_{1}}\left(e^{\lambda}\right)
$$




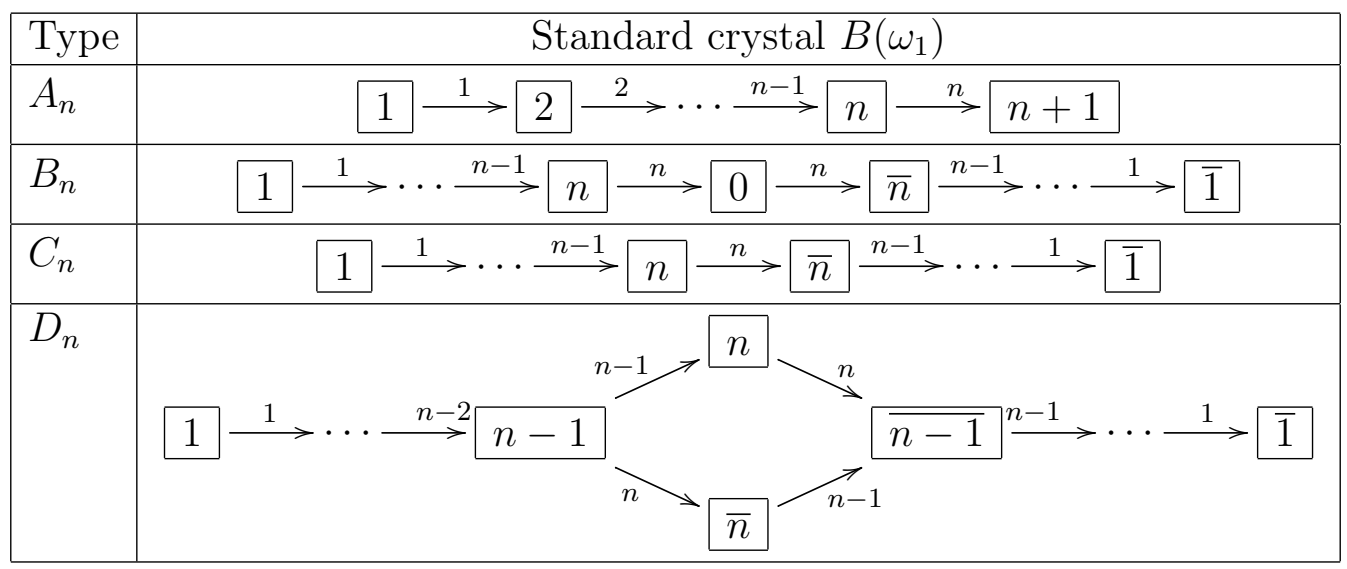

Figure 2: Standard crystals. The boxes represent the vertices of the crystal, and each arrow labeled $i$ shows the action of $f_{i}$.

\section{Kashiwara-Nakashima tableaux and branching rules}

\subsection{Kashiwara-Nakashima tableaux}

We briefly review a method, due to Kashiwara and Nakashima [33], of realizing all highest weight crystals of non-exceptional finite type $\mathfrak{g}$. The first observation is that many crystals occur inside high enough tensor powers of the "standard" crystals shown in Figure 2. Many here means all in type $A$ and $C$, but not those involving spin weights in types $B$ and $D$.

We call the set of symbols that show up in the boxes of the standard crystal of type $X_{n}=A_{n}, B_{n}, C_{n}, D_{n}$ the type $X_{n}$ alphabet. Impose a partial order $\prec$ on this alphabet by saying $x \prec y$ iff $x$ is to the left of $y$ in the presentation of the standard crystals in Figure 2 (in type $D_{n}$, the symbols $n$ and $\bar{n}$ are incomparable).

Definition 3.1. Fix $\mathfrak{g}$ of type $X_{n}$, for $X=A, B, C, D$. Fix a dominant integral weight $\gamma$ for $\mathfrak{g}=X_{n}$. Write $\gamma=m_{1} \omega_{1}+m_{2} \omega_{2}+\cdots+m_{n-1} \omega_{n-1}+m_{n} \omega_{n}$. Define a generalized partition $\Lambda(\gamma)$ associated to $\gamma$, which is defined case by case as follows:

- If $X=A, C, \Lambda(\gamma)$ has $m_{i}$ columns of height $i$ for each $1 \leqslant i \leqslant n$;

- If $X=B, \Lambda(\gamma)$ has $m_{i}$ columns of height $i$ for each $1 \leqslant i \leqslant n-1$, and $m_{n} / 2$ columns of height $n$;

- If $X=D, \Lambda(\gamma)$ has $m_{i}$ columns of each height $i$ for each $1 \leqslant i \leqslant n-2$, $\min \left(m_{n-1}, m_{n}\right)$ columns of height $n-1$, and $\left|m_{n}-m_{n-1}\right| / 2$ columns of height $n$. Color columns of height $n$ using color 1 if $m_{n}>m_{n-1}$ and color 2 if $m_{n}<m_{n-1}$.

We use French notation for partitions here, where we adjust the columns at the bottom. In cases where the above formulas involve a fractional number $x$ of columns at some height, we denote this by putting $\lfloor x\rfloor$ columns in addition to a single column of half width. Notice 


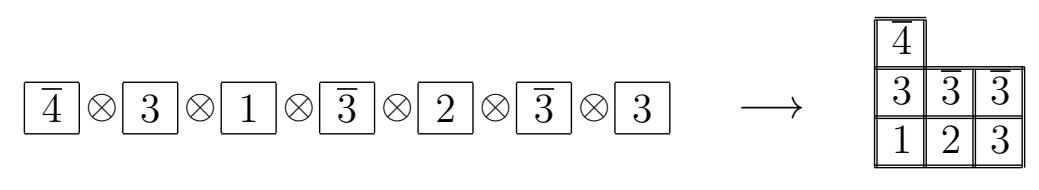

Figure 3: An element of $B\left(\omega_{3}+2 \omega_{2}\right)$ of type $C_{4}$ as realized inside the tensor product $B\left(\omega_{1}\right)^{\otimes 7}$ of seven copies of the standard crystal, and the corresponding tableau. For explicit conditions on which tableaux appear in this correspondence, see [33].

that this can only happen for columns of height $n$, and at worst we get a single column of width $1 / 2$.

Definition 3.2. Fix a dominant integral weight $\gamma$. We say $\gamma$ is a non-spin weight if

- In types $A_{n}$ and $C_{n}$, no conditions.

- In type $B_{n}, \Lambda(\gamma)$ does not contain any column of width $1 / 2$ (or equivalently, $m_{n}$ is even).

- In type $D_{n}, \Lambda(\gamma)$ has no columns of height $n$ (or equivalently, $m_{n-1}=m_{n}$ ).

In the case when $\Lambda=\Lambda(\gamma)$ does not contain a column of width $1 / 2$, the highest weight crystal $B(\gamma)$ embeds in the $M$-th tensor power of $B\left(\omega_{1}\right)$, where $M$ is the number of boxes in the partition $\Lambda$. Furthermore, the image of $B(\gamma)$ is contained in the set of $x_{M} \otimes \cdots \otimes x_{1}$ such, when entered into the Young diagram $\Lambda$ in order moving up columns and right to left (see Figure 3), the result is weakly increasing along rows and, if you ignore the symbols 0 in type $B$ and $n, \bar{n}$ in type $D$, also strictly increasing up columns. We refer to fillings which occur in the image of $B(\gamma)$ as Kashiwara-Nakashima (KN) tableaux. Precise conditions describing KN tableaux are given in [33]. The crystal structure on the set of KN tableaux is inherited from the crystal structure on $B\left(\omega_{1}\right)^{\otimes M}$. Modifications of this construction dealing with spin weights are given in [33].

Remark 3.3. The above construction of $B(\lambda)$ in type $D_{n}$ goes through without modification in the case $n=3$, when $D_{3} \cong A_{3}$. Thus we have two realizations of the crystal in this case.

\subsection{Branching rules and \pm diagrams}

We now describe branching rules for certain representations of $X_{n}$, where $X=B, C$ or $D$.

Definition 3.4. Fix a non-spin weight $\gamma$ (see Definition 3.2), and set $\Lambda=\Lambda(\gamma)$. A \pm diagram $P$ with outer shape $\Lambda$ is a sequence of partitions $\lambda \subseteq \mu \subseteq \Lambda$ such that $\Lambda / \mu$ and $\mu / \lambda$ are horizontal strips (i.e. every column contains at most one box). We depict a \pm diagram by filling $\mu / \lambda$ with the symbol + and those of $\Lambda / \mu$ with the symbol - . We make the additional type dependent modifications and restrictions: 
(i) In type $C_{n}, \lambda$ has no columns of height $n$.

(ii) In type $B_{n}, \lambda$ has no columns of height $n$. Additionally, we allow the \pm diagram to contain an extra symbol 0 . There can be at most one 0 , this must occur at height $n$, must be to the right of all + at height $n$, and to the left of all 0 at height $n$, and must be the only symbol in its column.

(iii) In type $D_{n}$, columns of $\lambda$ of height $n-1$ are either all colored 1 or all colored 2 .

We denote by outer $(P)=\Lambda$ the outer shape of $P$ and by $\operatorname{inner}(P)=\lambda$ the inner shape of $P$.

Embed $X_{n-1}$ into $X_{n}$ by removing node 1 from the Dynkin diagram of type $X_{n}$. In the special cases of $B_{2}, C_{2}$ and $D_{3}$, removing the node 1 gives a Dynkin diagram of a different type $\left(A_{1}, A_{1}\right.$, and $A_{1} \times A_{1}$, respectively). By abuse of notation, we use the symbol $X_{n-1}$ to mean this new diagram in these special cases. Although these special cases are not explicitly mentioned in [10], the proof of Theorem 3.5 goes through without change.

Theorem 3.5. (see [10, Section 3.2]) Fix a non-spin dominant integral weight $\gamma$ (see Definition 3.2) for $\mathfrak{g}=X_{n}$, and let $\Lambda=\Lambda(\gamma)$ (see Definition 3.1). Then there is a bijection between \pm diagrams $P$ with outer shape $\Lambda$ and $X_{n-1}$ highest weight elements in $B(\Lambda)$. This can be realized in terms of $K N$ tableaux by the following algorithm.

(i) For each + at height $n$, fill that column with $12 \ldots n$.

(ii) Replace each - with a $\overline{1}$ and, if there is a 0 in the \pm diagram, place a 0 in that position of the tableaux.

(iii) Fill the remainder of all columns by strings of the form $23 \ldots k$.

(iv) Let $S$ be the multi-set containing the heights of all the + in $P$ of height less than $n$. Move through the columns of $\Lambda$ from top to bottom, left to right, ignoring the columns $12 \ldots n$ of step (i), modifying the tableaux as follows. Each time you encounter a $\overline{1}$, replace it with $\overline{h+1}$, where $h$ is the largest element of $S$, and delete $h$ from $S$. Each time you encounter a 2 which is at the bottom of a column, replace the string $23 \ldots k$ in that column by $12 \ldots h h+2 \ldots k$, where $h$ is the largest element of $S$, and remove $h$ from $S$. Once $S$ is empty, stop.

(v) In type $D_{n}$, if the \pm diagram has empty columns of height $n-1$ colored 2 , change all occurrences of $n$ at height $n-1$ to $\bar{n}$.

Furthermore, two \pm diagrams $P$ and $P^{\prime}$ correspond to $X_{n-1}$ highest weight vectors of the same $X_{n-1}$ weight if and only if inner $(P)=\operatorname{inner}\left(P^{\prime}\right)$. 
Remark 3.6. Definition 3.4 part (iii) differs from the statement in [10], since in that paper there were no colorings for columns of height $n-1$. These colorings are needed, as can be seen by considering the branching rules for $B\left(\omega_{n-1}+\omega_{n}\right)$ for $D_{4}$ :
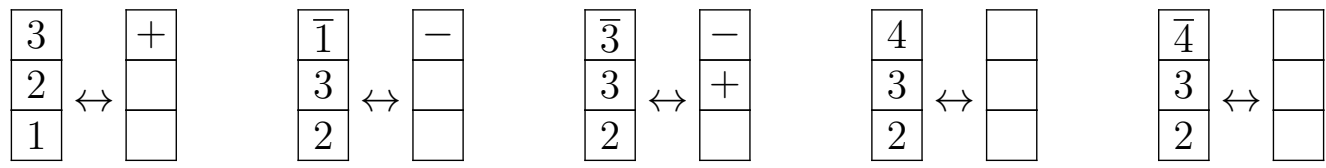

where the fourth \pm diagram is considered of color 1 and the fifth of color 2 .

Definition 3.7. Let $\gamma$ be an integral highest weight of type $X_{n}$ and write $\gamma=m_{1} \omega_{1}+$ $m_{2} \omega_{2}+\cdots+m_{n-1} \omega_{n-1}+m_{n} \omega_{n}$. We call $\gamma$ a case (AUT) weight if it satisfies:

- If $X=C$, no conditions;

- If $X=B$, assume $m_{n}=0$;

- If $X=D, m_{n}=m_{n-1}=0$.

Note that in all these cases, $\Lambda(\gamma)$ is an ordinary partition.

Remark 3.8. The term "case (AUT)" in Definition 3.7 is motivated by the fact that these are exactly the classical highest weights that appear in case (AUT) KR crystals, as defined in Section 4 below.

Remark 3.9. All case (AUT) weights are non-spin, as in Definition 3.2. Furthermore, if one starts with a case (AUT) weight $\gamma$, then the $X_{n-1}$ weights which appear in the decomposition from Theorem 3.5 will all be non-spin weights, and the $X_{n-1}$ highest weight $\gamma^{\prime}$ corresponding to the \pm diagram $P$ will satisfy $\Lambda\left(\gamma^{\prime}\right)=\operatorname{inner}(P)$.

\subsection{Nested \pm diagrams}

If $\gamma$ is a case (AUT) weight, $n \geqslant 3$ in type $B_{n}, C_{n}$, and $n \geqslant 4$ in type $D_{n}$, it follows immediately from Section 3.2 that the branching rule from $X_{n}$ to $X_{n-2}$ can be described using pairs of \pm diagrams $P$ and $p$ with outer $(P)=\Lambda(\gamma)$ and inner $(P)=\operatorname{outer}(p)$. We call such pairs of \pm diagrams nested. The following result from [55] gives an explicit description of the action of $e_{1}$ on an $X_{n-2}$ highest weight vector in terms of pairs of \pm diagrams. Since $e_{1}$ commutes with all $e_{j}$ for $j \geqslant 3$, this completely describes $e_{1}$.

Pair off the symbols in $(P, p)$ according to the rules

- Successively run through all + in $p$ from left to right and, if possible, pair it with the leftmost yet unpaired + in $P$ weakly to the left of it.

- Successively run through all - in $p$ from left to right and, if possible, pair it with the rightmost yet unpaired - in $P$ weakly to the left.

- Successively run through all yet unpaired + in $p$ from left to right and, if possible, pair it with the leftmost yet unpaired - in $p$. 
Notice that a \pm diagram is uniquely determined by its outer (or inner) shape along with the number of each symbol,+- , and 0 on each row. Similarly, a pair $(P, p)$ of nested \pm diagrams is uniquely determined by its intermediate shape $\operatorname{int}(P, p)=\operatorname{outer}(p)=$ inner $(P)$, along with the data of how many of each symbol is in each row of $P$ and of $p$. Since $e_{1}$ commutes with $e_{j}$ for all $j \geqslant 3$, the following gives a complete description of the action of $e_{1}$ on the crystal:

Lemma 3.10. [55, Lemma 5.1] Let $\gamma$ be a case (AUT) weight and fix a pair $(P, p)$ of nested \pm diagrams such that outer $(P)=\Lambda(\gamma)$. Let $b$ be the corresponding $X_{n-2}$ highest weight vector in $B(\gamma)$. If there are no unpaired + in $p$ and no unpaired - in $P$, then $e_{1}(b)=0$. Otherwise, $e_{1}(b)$ is the $X_{n-2}$ highest weight element corresponding to the pair of \pm diagrams $\left(P^{\prime}, p^{\prime}\right)$ described as follows.

If there is an unpaired + in $p$, let $k$ be the height the rightmost unpaired + in $p$.

- If there is a-directly above the chosen + in $p$, then int $(P, p) / \operatorname{int}\left(P^{\prime}, p^{\prime}\right)$ is a single box at height $k+1$. All rows of $P$ and $p$ have the same number of each symbol except: There is one more + in $P$ at height $k+1$; there is one less - in $p$ at height $k+1$; there is one less + and one more - in $p$ at height $k$.

- Otherwise, $\operatorname{int}(P, p) / \operatorname{int}\left(P^{\prime}, p^{\prime}\right)$ is a single box at height $k$. All rows of $P$ and $p$ have the same number of each symbol except: There is one more + in $P$ at height $k$; there is one less + in $p$ at height $k$.

Otherwise let $k$ be the height of the leftmost unpaired - in P.

- If there is a + directly below the chosen - in $P$, then int $\left(P^{\prime}, p^{\prime}\right) / \operatorname{int}(P, p)$ is a single box at height $k-1$. All rows of $P$ and $p$ have the same number of each symbol except: There is one less - and one more + in $P$ at height $k$; there is one less + in $P$ at height $k-1$; there is one more - in $p$ at height $k-1$.

- Otherwise, $\operatorname{int}\left(P^{\prime}, p^{\prime}\right) / \operatorname{int}(P, p)$ is a single box at height $k$. All rows of $P$ and $p$ have the same number of each symbol except: There is one less - in $P$ at height $k$; there is one more - in $p$ at height $k$.

In type $B$, in every case, the number of $0 s$ in $p$ remains unchanged ( $P$ cannot contain 0 since we are assuming that $\gamma$ is in case (AUT)).

Remark 3.11. In principle, one could build a new combinatorial model for type $B_{n}$, $C_{n}, D_{n}$ crystals using nested \pm diagrams, where the crystal operators would be given by Lemma 3.10. That is, the tableaux would consist of $n-1$ nested \pm diagrams in type $B_{n}, C_{n}$ and $n-2$ nested \pm diagrams in type $D_{n}$, along with some extra data recording an element in a representation of $A_{1}$ (or $A_{1} \times A_{1}$ in type $D_{n}$ ). This would have some advantages over KN tableaux, in that branching rules would be more readily visible. Such tableaux may also be more convenient for working with KR crystals. 


\section{Realizations of KR crystals}

Explicit combinatorial models for $\mathrm{KR}$ crystals $B^{r, s}$ for the non-exceptional types were constructed in [10]. In this section we recall and prove some properties that we need for the definition of the energy function in Section 5 and Lemma 7.2.

The results are presented for three different cases:

(IRR) The classically irreducible $\mathrm{KR}$ crystals $B^{r, s}$ :

$$
\begin{array}{ll}
A_{n}^{(1)} & 1 \leqslant r \leqslant n \\
D_{n}^{(1)} & r=n-1, n \\
D_{n+1}^{(2)} & r=n \\
C_{n}^{(1)} & r=n
\end{array}
$$

(AUT) KR crystals $B^{r, s}$ constructed via Dynkin automorphisms:

$$
\begin{array}{ll}
D_{n}^{(1)} & 1 \leqslant r \leqslant n-2 \\
B_{n}^{(1)} & 1 \leqslant r \leqslant n-1 \\
A_{2 n-1}^{(2)} & 1 \leqslant r \leqslant n
\end{array}
$$

(VIR) The remaining virtually constructed KR crystals:

$$
\begin{array}{ll}
B_{n}^{(1)} & B^{n, s} \\
C_{n}^{(1)} & B^{r, s} \text { for } 1 \leqslant r<n \\
D_{n+1}^{(2)} & B^{r, s} \text { for } 1 \leqslant r<n \\
A_{2 n}^{(2)} & B^{r, s} \text { for } 1 \leqslant r \leqslant n .
\end{array}
$$

In Section 4.1 we present the classical decomposition of the KR crystals in the various cases. Case (IRR) is discussed in Section 4.2. This case is well-understood, and we simply state the facts we need. Case (AUT) is handled in Section 4.3. This time we discuss the realizations in more detail, and prove a technical lemma. Case (VIR) is handled in Sections 4.4, 4.5 and 4.6. Following [10], we realize these KR crystals as virtual crystals, using a combination of the similarity method of Kashiwara [29] and the technique of virtual crystals developed in $[51,52,10]$. We refer to crystals obtained using both of these methods as virtual crystals.

\subsection{Classical decompositions of KR crystals}

Set

$$
\diamond= \begin{cases}\emptyset & \text { for type } A_{n}^{(1)} \text { and } 1 \leqslant r \leqslant n \\ & \text { for types } C_{n}^{(1)}, D_{n+1}^{(2)} \text { and } r=n \\ & \text { for type } D_{n}^{(1)} \text { and } r=n-1, n \\ \text { vertical domino } & \text { for type } D_{n}^{(1)} \text { and } 1 \leqslant r \leqslant n-2 \\ & \text { for types } B_{n}^{(1)}, A_{2 n-1}^{(2)} \text { and } 1 \leqslant r \leqslant n \\ \text { horizontal domino } & \text { for types } C_{n}^{(1)}, D_{n+1}^{(2)} \text { and } 1 \leqslant r<n \\ \text { box } & \text { for type } A_{2 n}^{(2)} \text { and } 1 \leqslant r \leqslant n .\end{cases}
$$


Then every $B^{r, s}$ decomposes as a classical crystal as

$$
B^{r, s} \cong \bigoplus_{\lambda} B(\lambda)
$$

where the sum is over those $\lambda$ such that $\Lambda(\lambda)$ (see Definition 3.1) can be obtained from the rectangle $\Lambda\left(s \omega_{r}\right)$ by removing some number of $\diamond$, each occurring with multiplicity 1 . In the untwisted case this was obtained by Chari [3]. In the twisted case, it was conjectured in [16, Appendix A] and proven by Hernandez (see [19, Section 5]).

\subsection{Classically irreducible KR crystals (case (IRR))}

As a $\{1,2, \ldots, n\}$-crystal, $B^{r, s} \cong B\left(r \omega_{s}\right)$. For example, the realization of the $\mathrm{KR}$ crystal $B^{r, s}$ of type $A_{n}^{(1)}$ is well-known in terms of rectangular Young tableaux of shape $\left(s^{r}\right)$. We refer the reader to e.g. [10, Section 4.1] for details. The construction of the other irreducible KR crystals can be found in [10, Section 6]. We only need the following fact, which follows immediately from the explicit models.

Lemma 4.1. Let $B^{r, s}$ be a KR crystal of type $\mathfrak{g}$ for one of the cases in case (IRR). Then for all $b \in B^{r, s}, \varepsilon_{0}(b) \leqslant s$.

\subsection{KR crystals constructed via Dynkin automorphisms (case $(\mathrm{AUT}))$}

Let $\mathfrak{g}$ be of type $D_{n}^{(1)}, B_{n}^{(1)}$ or $A_{2 n-1}^{(2)}$, with the underlying finite type Lie algebra of type $X_{n}=D_{n}, B_{n}$ or $C_{n}$, respectively. Fix $s>0$ and $r$ so that $B^{r, s}$ is in case (AUT). Consider the classical crystal

$$
C^{r, s}:=\bigoplus B(\lambda)
$$

where the sum is over all $\lambda$ such that $\Lambda(\lambda)$ (see Definition 3.1) can be obtained from $\Lambda\left(s \omega_{r}\right)$ by removing vertical dominos. As in Section 3.2, the $X_{n-1}$ highest weight elements in $C^{r, s}$ (i.e. the highest weight element for the algebra with Dynkin diagram $I \backslash\{0,1\}$ ) are indexed by \pm diagrams whose outer shape can be obtained from $\Lambda\left(s \omega_{r}\right)$ by removing vertical dominos.

Definition 4.2. Define the involution $\varsigma$ on the $X_{n-1}$ highest weight vectors of $C^{r, s}$, as indexed by \pm diagrams, as follows. Let $P$ be a \pm diagram with $\operatorname{outer}(P)=\Lambda$ and $\operatorname{inner}(P)=\lambda$. For each $1 \leqslant i \leqslant r-1$ :

(i) If $i \equiv r-1(\bmod 2)$ then above each column of $\lambda$ of height $i$, there must be a + or a - . Interchange the number of such + and - .

(ii) If $i \equiv r(\bmod 2)$ then above each column of $\lambda$ of height $i$, either there are no signs or a $\mp$ pair. Interchange the number of columns of each type.

By Theorem 3.5, this can be extended in a unique way to an involution $\varsigma$ on $C^{r, s}$. 
Theorem 4.3. $[55,10]$ Fix $\mathfrak{g}$ and a KR crystal $B^{r, s}$ in case (AUT). Define operators $e_{0}$ and $f_{0}$ on $C^{r, s}$ by $e_{0}:=\varsigma \circ e_{1} \circ \varsigma$ and $f_{0}:=\varsigma \circ f_{1} \circ \varsigma$. Then $C^{r, s}$ along with these new operators is isomorphic to $B^{r, s}$.

Lemma 4.4. Fix $\mathfrak{g}$ and a $K R$ crystal $B^{r, s}$ as in case (AUT). Consider the realization of $B^{r, s}$ given in Theorem 4.3. Fix $b \in B^{r, s}$, and assume $b$ lies in the component $B(\gamma)$ and $e_{0}(b)$ lies in the classical component $B\left(\gamma^{\prime}\right)$. Then

(i) $\Lambda\left(\gamma^{\prime}\right)$ is either equal to $\Lambda(\gamma)$, or else is obtained from $\Lambda(\gamma)$ by adding or removing a single vertical domino.

(ii) If $\varepsilon_{0}(b)>s$, then $\Lambda\left(\gamma^{\prime}\right)$ is obtained from $\Lambda(\gamma)$ by removing a vertical domino.

Proof. Recall that for $b \in B^{r, s}$ by definition $e_{0}(b)=\varsigma \circ e_{1} \circ \varsigma(b)$. Since $e_{0}$ and $e_{1}$ commute with $e_{i}$ for $i=3,4, \ldots, n$, they are defined on $X_{n-2}$ components, which are described by pairs of \pm diagrams $(P, p)$. Consider the 1 -string $\left\{b_{0}, b_{1}, \ldots, b_{k}\right\}$ where $b_{i}=e_{1}^{i} \circ \varsigma(b)$ with corresponding \pm diagrams $\left(P_{i}, p_{i}\right)$. By Lemma 3.10 there exists a $0 \leqslant j \leqslant k$ such that:

(a) For $0 \leqslant i<j, P_{i+1}$ is obtained from $P_{i}$ by the addition of one box containing + ; the + are added from right to left with increasing $i$.

(b) For $j \leqslant i<k, P_{i+1}$ is obtained from $P_{i}$ by the removal of one box containing - ; the - are removed from left to right with increasing $i$.

Recall that $\varsigma$ interchanges + and - in columns of height congruent to $1(\bmod r)$ and F-pairs and empty columns of height congruent to $0(\bmod r)$. Empty columns of height $r$ are left unchanged. Since the width of the diagrams is at most $s$, we can only have $\varepsilon_{0}(b)>s$ if we are in case (a) above and the + is added at height strictly smaller than $r$. Then there are two cases:

- The + is added below a - in $P_{0}$.

- The + is added in an empty column in $P_{0}$.

In both cases it is easy to check from the rules of $\varsigma$ that a vertical domino is removed from the outer shape.

In all other cases, applying $e_{1}$ to $\varsigma(b)$ only adds or removes a single symbol to/from the corresponding \pm diagram $P$, so from the definition of $\varsigma$ and the fact that $e_{0}=\varsigma \circ e_{1} \circ \varsigma$, it is clear that $\Lambda\left(\gamma^{\prime}\right)$ differs from $\Lambda(\gamma)$ by at most one vertical domino, and the lemma holds.

\section{4 $B^{r, s}$ of type $C_{n}^{(1)}$ for $r<n$ (in case (VIR))}

The KR crystals of type $C_{n}^{(1)}$ are constructed inside an ambient crystal of type $A_{2 n+1}^{(2)}$. 
Theorem 4.5. [10, Section 4.3, Theorem 5.7] Fix $n \geqslant 2$ and $r<n$. Let $\hat{B}^{r, s}$ be the $K R$ crystal corresponding to type $A_{2 n+1}^{(2)}$, with crystal operators $\hat{e}_{0}, \ldots, \hat{e}_{n+1}$. Let $V$ be the subset of all $b \in \hat{B}^{r, s}$ which are invariant under the involution $\varsigma$ from Definition 4.2. Define the virtual crystal operators $e_{i}:=\hat{e}_{i+1}$ for $1 \leqslant i \leqslant n$ and $e_{0}:=\hat{e}_{0} \hat{e}_{1}$. Then $V$ along with the operators $e_{0}, \ldots, e_{n}$ is isomorphic to $B^{r, s}$ for type $C_{n}^{(1)}$.

Here the operators $f_{i}$ and $\hat{f}_{i}$ are defined by the condition $f_{i}(b)=b^{\prime}$ if and only if $e_{i}\left(b^{\prime}\right)=b$.

Recall that as a classical crystal, $B^{r, s}$ of type $C_{n}^{(1)}$ decomposes as in (4.2).

Lemma 4.6. Let $B^{r, s}$ with $r<n$ be the KR crystal of type $C_{n}^{(1)}$. Fix $b \in B^{r, s}$, and assume $b$ lies in the component $B(\gamma)$ and $e_{0}(b)$ lies in the classical component $B\left(\gamma^{\prime}\right)$. Then

(i) $\Lambda\left(\gamma^{\prime}\right)$ is either equal to $\Lambda(\gamma)$, or else is obtained from $\Lambda(\gamma)$ by adding or removing a single horizontal domino.

(ii) If $\varepsilon_{0}(b)>\lceil s / 2\rceil$, then $\Lambda\left(\gamma^{\prime}\right)$ is obtained from $\Lambda(\gamma)$ by removing a horizontal domino.

Proof. Realize the crystal $B^{r, s}$ of type $C_{n}^{(1)}$ inside the ambient crystal $\hat{B}^{r, s}$ of type $A_{2 n+1}^{(2)}$ using Theorem 4.5. Denote the embedding by $S: B^{r, s} \hookrightarrow \hat{B}^{r, s}$, and set $\hat{b}=S(b)$. Let $(P, p)$ be the pair of \pm diagrams associated to the $\{3,4, \ldots, n+1\}$ highest weight corresponding to $\hat{b}$, and let $\gamma$ be the highest weight of $b$ as a $C_{n} \subset C_{n}^{(1)}$ crystal. Since $e_{0}=\hat{e}_{1} \hat{e}_{0}$ and $e_{i}=\hat{e}_{i+1}, \Lambda(\gamma)=\operatorname{inner}(P)$.

Recall from [10, Section 4.3] that $S(b)$ is invariant under $\varsigma$, hence in particular $P$ is invariant under $\varsigma$. Also, as operators on $\hat{B}^{r, s}$,

$$
S\left(e_{0}\right)=\hat{e}_{1} \hat{e}_{0}=\hat{e}_{1} \circ \varsigma \circ \hat{e}_{1} \circ \varsigma=\hat{e}_{1} \circ \varsigma \circ \hat{e}_{1} .
$$

By Lemma 3.10, $\hat{e}_{1}$ either moves a + from $p$ to $P$, or moves a - from $P$ to $p$. That is, outer $(P)$ is unchanged, inner $(P)$ only changes by a single box, and, except for the new + or missing - , the number of + and - on each row of $P$ is unchanged. Also, in all situations $\varsigma$ preserves inner $(P)$. Thus (4.3) implies that inner $(P)$ can change by at most 2 boxes. This along with the description of the classical decomposition of $B^{r, s}$ implies part (i).

Now assume that $\varepsilon_{0}(b)>\lceil s / 2\rceil$. Since $P$ is $s$-invariant, it is clear that there can be at most $\lfloor s / 2\rfloor$ symbols - in $P$. Since $\varepsilon_{0}(b)=\hat{\varepsilon}_{1}(\hat{b})>s / 2$, by the description of $\hat{e}_{1}$ from Lemma 3.10 we see that $\hat{e}_{1}$ must move a from $p$ to $P$. There are three cases:

(a) The + is added below a - in $P$.

(b) The + is added in an empty column in $P$ at height less than $r$.

(c) The + is added in an empty column in $P$ at height $r$. 
It is not hard to check that in case $(\mathrm{a})$, in $\varsigma \circ \hat{e}_{1}(P)$, there is one less + in row $k+1$ and one more empty column of height $k-1$ compared to $P$, and everything else is unchanged. Since both $P$ and $\hat{e}_{1} \circ \varsigma \circ \hat{e}_{1}(P)$ are invariant under $\varsigma$, the only possibility is that the final $\hat{e}_{1}$ must add another + to row $k$. Thus two symbols have been added to $P$ in row $k$, which has the effect of removing a horizontal domino from inner $(P)$.

In case $(\mathrm{b})$, in $\varsigma \circ \hat{e}_{1}(P)$, there is one less $\mp$-pair in row $k+1$ and one more - in row $k$ and everything else is unchanged. Again since $\hat{e}_{1} \circ \varsigma \circ \hat{e}_{1}(b)$ has to be invariant under $\varsigma$, the only possibility is that $\hat{e}_{1}$ adds another + to row $k$ of $\varsigma \circ \hat{e}_{1}(P)$. Thus $S\left(e_{0}\right)$ removes a horizontal domino from $\operatorname{inner}(P)$.

In case (c), $P$ has at least one column with no symbols, and so $P$ contains at most $\lfloor(s-1) / 2\rfloor$ symbols - . Since $\hat{\varepsilon}_{1}(\hat{b})=\varepsilon_{0}(b)>\lceil s / 2\rceil$, there are at least 2 uncanceled + in $p$. Consider the double \pm diagram $\left(P^{\prime}, p^{\prime}\right)$ for $\varsigma \circ \hat{e}_{1}(\hat{b})$. This differs from $(P, p)$ by: $P^{\prime}$ has an extra - at height $r$, and $p^{\prime}$ has one less + . Looking at the cancelation rules before Lemma 3.10, there is still an uncanceled + in $p^{\prime}$ (corresponding to the second uncanceled + in $p$ ). Thus the second $\hat{e}_{1}$ moves another + into $P^{\prime}$. So in total $S\left(e_{0}\right)=\hat{e}_{1} \circ \varsigma \circ \hat{e}_{1}$ decreases the inner shape of $P$ by 2 boxes, which must remove a horizontal domino.

\section{5 $B^{n, s}$ in type $B_{n}^{(1)}$ (in case (VIR))}

Theorem 4.7. (see [10, Lemma 4.2]) Let $\hat{B}^{n, s}$ be the KR crystal of type $A_{2 n-1}^{(2)}$ with crystal operators $\hat{e}_{0}, \ldots, \hat{e}_{n}$. Let $V$ be the subset of $\hat{B}^{n, s}$ which can be reached from the classical highest weight element in $\hat{B}^{n, s}$ of weight $s \omega_{n}$ using the virtual crystal operators

$e_{i}=\hat{e}_{i}^{2}$ for $0 \leqslant i \leqslant n-1, e_{n}=\hat{e}_{n}$. Then $V$ along with the operators $e_{0}, \ldots, e_{n}$ is isomorphic to $B^{n, s}$ for $B_{n}^{(1)}$.

Furthermore, the type $B_{n}$ highest weight vectors in $V$ are exactly the type $C_{n}$ highest weight vectors in $\hat{B}^{n, s}$ that can be obtained from $\Lambda\left(s \omega_{r}\right)$ by removing $2 \times 2$ blocks.

Proof. The virtual crystal realization is proven in [10, Lemma 4.2]. The classical decomposition of $B^{n, s}$ as a type $B_{n}$ crystal is

$$
\bigoplus_{\mathbf{k}} B\left(k_{\iota} \omega_{\iota}+k_{\iota+2} \omega_{\iota+2}+\cdots+k_{n-2} \omega_{n-2}+k_{n} \omega_{n}\right)
$$

where $\iota=0,1$ so that $\iota \equiv n(\bmod 2), \omega_{0}=0$, and the sum is over all nonnegative integer vectors $\mathbf{k}$ such that $2 k_{\iota}+\cdots+2 k_{n-2}+k_{n}<s$. As discussed in [10, Lemma 4.2], the highest weight vectors for each of these components must be classical type $C_{n}$ highest weight vector in the ambient crystal $\hat{B}^{n, s}$ of weight

$$
2 k_{\iota} \omega_{\iota}+2 k_{\iota+2} \omega_{\iota+2}+\cdots+2 k_{n-2} \omega_{n-2}+k_{n} \omega_{n} .
$$

The weight of these highest weight vectors in the ambient crystal are exactly those $\gamma$ such that $\Lambda(\gamma)$ is obtained from $\Lambda\left(s \omega_{r}\right)$ by removing $2 \times 2$ blocks. The result follows.

Lemma 4.8. Let $B^{n, s}$ be the KR crystal of type $B_{n}^{(1)}$. Fix $b \in B^{n, s}$, and assume $b$ lies in the component $B(\gamma)$ and $e_{0}(b)$ lies in the classical component $B\left(\gamma^{\prime}\right)$. Then 
(i) $\Lambda\left(\gamma^{\prime}\right)$ is either equal to $\Lambda(\gamma)$, or else is obtained from $\Lambda(\gamma)$ by adding or removing a single vertical domino.

(ii) If $\varepsilon_{0}(b)>\lceil s / 2\rceil$, then $\Lambda\left(\gamma^{\prime}\right)$ is obtained from $\Lambda(\gamma)$ by removing a vertical domino.

Proof. For part (i), use the fact that by the virtual realization from Theorem 4.7, $e_{0}=\hat{e}_{0}^{2}$. So, it follows from Lemma 4.4 that $e_{0}$ adds or subtracts at most 2 vertical dominoes. By Theorem 4.7, the only possibilities are that $e_{0}$ either leaves the shape unchanged, or adds or subtracts a single $2 \times 2$ block.

Part (ii) follows since, if $\varepsilon_{0}(b)>\lceil s / 2\rceil$, then $\hat{\varepsilon}_{0}(b) \geqslant s+2$. Hence applying $e_{0}=\hat{e}_{0}^{2}$ subtracts 2 vertical dominoes by Lemma 4.4, which as above must fit together as a $2 \times 2$ block.

\subsection{The KR crystals of type $A_{2 n}^{(2)}$ and $D_{n+1}^{(2)}$ in case (VIR)}

We now present the virtual crystal construction of the KR crystals $B^{r, s}$ of types $A_{2 n}^{(2)}$ and $D_{n+1}^{(2)}$ of case (VIR), and then prove the analogue of Lemma 4.4 in this setting.

Theorem 4.9. [10, Section 4.4, Theorem 5.7] Fix $n \geqslant 2$. Let $\hat{B}^{r, 2 s}$ be the KR crystal corresponding to type $C_{n}^{(1)}$, with crystal operators $\hat{e}_{0}, \ldots, \hat{e}_{n}$. In each case below, let $V$ be the subset of $\hat{B}^{r, 2 s}$ which can be reached from the classical highest weight element in $\hat{B}^{r, 2 s}$ of weight $2 s \omega_{r}$ using the listed virtual crystal operators:

(i) Let $1 \leqslant r<n$. Define $e_{i}:=\hat{e}_{i}^{2}$ for $1 \leqslant i \leqslant n-1, e_{0}:=\hat{e}_{0}$, and $e_{n}:=\hat{e}_{n}$. Then $V$, along with the operators $e_{0}, \ldots, e_{n}$, is isomorphic to the KR crystal $B^{r, s}$ for type $D_{n+1}^{(2)}$.

(ii) Let $1 \leqslant r \leqslant n$. Define $e_{i}:=\hat{e}_{i}^{2}$ for $1 \leqslant i \leqslant n$ and $e_{0}:=\hat{e}_{0}$. Then $V$ along with the operators $e_{0}, \ldots, e_{n}$ is $B^{r, s}$ for type $A_{2 n}^{(2)}$.

Here the operators $f_{i}$ and $\hat{f}_{i}$ are defined by the condition $f_{i}(b)=b^{\prime}$ if and only if $e_{i}\left(b^{\prime}\right)=b$.

Lemma 4.10. Consider $B^{r, s}$ of type $D_{n+1}^{(2)}$ with $r<n$, or of type $A_{2 n}^{(2)}$ with $1 \leqslant r \leqslant n$. Fix $b \in B^{r, s}$, and assume $b$ lies in the classical (type $B_{n}$ or $C_{n}$, respectively) component $B(\gamma)$, and $e_{0}(b)$ lies in the classical component $B\left(\gamma^{\prime}\right)$. Then

(i) $\Lambda\left(\gamma^{\prime}\right)$ is either equal to $\Lambda(\gamma)$, or else is obtained from $\Lambda(\gamma)$ by adding or removing a single box.

(ii) If $\varepsilon_{0}(b)>s$, then $\Lambda\left(\gamma^{\prime}\right)$ is obtained from $\Lambda(\gamma)$ by removing a box.

Proof. Let $\hat{B}^{r, 2 s}$ be the ambient KR crystal of type $C_{n}^{(1)}$ with crystal operators $\hat{e}_{i}$ as in Theorem 4.9. Denote the virtualization map $S: B^{r, s} \rightarrow V \subseteq \hat{B}^{r, 2 s}$. Recall that $V$ is the subset of $\hat{B}^{r, 2 s}$ generated by the element of weight $2 s \omega_{r}$ by applying the virtual crystal operators $\hat{e}_{i}$ :

(i) In type $D_{n+1}^{(2)}, e_{0}=\hat{e}_{0}, e_{n}=\hat{e}_{n}$, and $e_{i}=\hat{e}_{i}^{2}$ for $1 \leqslant i \leqslant n-1$. 
(ii) In type $A_{2 n}^{(2)}, e_{0}=\hat{e}_{0}$, and $e_{i}=\hat{e}_{i}^{2}$ for $1 \leqslant i \leqslant n$.

By Lemma 4.6, $\hat{e}_{0}$ changes the classical component of the underlying KR crystal of type $C_{n}^{(1)}$ by adding or subtracting at most one horizontal domino, and furthermore if $\hat{\varepsilon}_{0}(S(b))>s$, then $\hat{e}_{0}$ always removes a horizontal domino. The result now follows immediately from the description of the classical components of these virtual crystals as given in [10, Lemma 4.9].

Note that in the case of $A_{2 n}^{(2)}$ with $r=n, \hat{B}^{n, 2 s}$ is not a KR crystal of type $C_{n}^{(1)}$. However, Theorem 4.5 still gives a type $C_{n}^{(1)}$ combinatorial crystal in this case and Lemma 4.6 still holds. So the proof still goes though in this case.

\section{$5 \quad$ Energy functions}

We define two energy functions on tensor products of $\mathrm{KR}$ crystals. The function $E^{\text {int }}$ is given by a fairly natural "global" condition on tensor products of level- $\ell \mathrm{KR}$ crystals. The function $D$ is defined by summing up combinatorially defined "local" contributions, and makes sense for general tensor products of KR crystals. It was suggested (but not proven) in [52, Section 2.5] that, when $E^{\text {int }}$ is defined, these two functions agree up to a shift. This will be proven in Theorem 7.5 below.

\subsection{The function $E^{\text {int }}$}

Definition 5.1. For each node $r \in I \backslash\{0\}$ and each $\ell \in \mathbb{Z}_{>0}$, let $u_{r, \ell c_{r}}$ be the unique element of $B^{r, \ell c_{r}}$ such that $\varepsilon\left(u_{r, \ell c_{r}}\right)=\ell \Lambda_{0}$ (which exists as $B^{r, \ell c_{r}}$ is perfect).

The following is essentially the definition of a ground state path from [25].

Definition 5.2. Let $B=B^{r_{N}, \ell c_{r_{N}}} \otimes \cdots \otimes B^{r_{1}, \ell c_{r_{1}}}$ be a composite level- $\ell \mathrm{KR}$ crystal. Define $u_{B}=u_{B}^{N} \otimes \cdots \otimes u_{B}^{1}$ to be the unique element of $B$ such that

(i) $u_{B}^{1}=u_{r_{1}, \ell c_{r_{1}}}$ and

(ii) for each $1 \leqslant j<N, \varepsilon\left(u_{B}^{j+1}\right)=\varphi\left(u_{B}^{j}\right)$.

This $u_{B}$ is well-defined by condition (v) in Definition 2.4 of a perfect crystal. The element $u_{B}$ is called the ground state path of $B$.

Definition 5.3. Let $B$ be a composite KR crystal of level $\ell$ and consider $u_{B}$ as in Definition 5.2. The intrinsic energy function $E^{\text {int }}$ on $B$ is defined by setting $E^{\text {int }}(b)$ to be the minimal number of $f_{0}$ in a string $f_{i_{N}} \cdots f_{i_{1}}$ such that $f_{i_{N}} \cdots f_{i_{1}}\left(u_{B}\right)=b$. 


\subsection{The $D$ function}

Definition 5.4. The $D$-function on $B^{r, s}$ is the function defined as follows:

(i) $D_{B^{r, s}}: B^{r, s} \rightarrow \mathbb{Z}$ is constant on all classical components.

(ii) On the component $B(\lambda), D_{B^{r, s}}$ records the maximum number of $\diamond$ that can be removed from $\Lambda(\lambda)$ such that the result is still a (generalized) partition, where $\diamond$ is as in (4.1).

In those cases when $\diamond=\emptyset$, this is interpreted as saying that $D_{B^{r, s}}$ is the constant function 0 .

Let $B_{1}, B_{2}$ be two affine crystals with generators $v_{1}$ and $v_{2}$, respectively, such that $B_{1} \otimes B_{2}$ is connected and $v_{1} \otimes v_{2}$ lies in a one-dimensional weight space. By [39, Proposition 3.8 , this holds for any two KR crystals. The generator $v$ for the $\mathrm{KR}$ crystal $B^{r, s}$ is chosen to be the unique element of classical weight $s \omega_{r}$.

The combinatorial R-matrix [25, Section 4] is the unique crystal isomorphism

$$
\sigma: B_{2} \otimes B_{1} \rightarrow B_{1} \otimes B_{2} .
$$

By weight considerations, this must satisfy $\sigma\left(v_{2} \otimes v_{1}\right)=v_{1} \otimes v_{2}$.

As in [25] and [51, Theorem 2.4], there is a function $H=H_{B_{2}, B_{1}}: B_{2} \otimes B_{1} \rightarrow \mathbb{Z}$, unique up to global additive constant, such that, for all $b_{2} \in B_{2}$ and $b_{1} \in B_{1}$,

$$
H\left(e_{i}\left(b_{2} \otimes b_{1}\right)\right)=H\left(b_{2} \otimes b_{1}\right)+ \begin{cases}-1 & \text { if } i=0 \text { and LL } \\ 1 & \text { if } i=0 \text { and RR } \\ 0 & \text { otherwise. }\end{cases}
$$

Here LL (resp. RR) indicates that $e_{0}$ acts on the left (resp. right) tensor factor in both $b_{2} \otimes b_{1}$ and $\sigma\left(b_{2} \otimes b_{1}\right)$. When $B_{1}$ and $B_{2}$ are KR crystals, we normalize $H_{B_{2}, B_{1}}$ by requiring $H_{B_{2}, B_{1}}\left(v_{2} \otimes v_{1}\right)=0$, where $v_{1}$ and $v_{2}$ are the generators defined above.

Definition 5.5. For $B=B^{r_{N}, s_{N}} \otimes \cdots \otimes B^{r_{1}, s_{1}}, 1 \leqslant i \leqslant N$ and $i<j \leqslant N$, set

$$
D_{i}:=D_{B^{r_{i}, s_{i}}} \sigma_{1} \sigma_{2} \cdots \sigma_{i-1} \quad \text { and } \quad H_{j, i}:=H_{i} \sigma_{i+1} \sigma_{i+2} \cdots \sigma_{j-1},
$$

where $\sigma_{i}$ and $H_{i}$ act on the $i$-th and $(i+1)$-st tensor factors and $D_{B^{r_{i}, s_{i}}}$ is the $D$-function on the rightmost tensor factor $B^{r_{i}, s_{i}}$ as given in Definition 5.4. The $D$-function $D_{B}: B \rightarrow \mathbb{Z}$ is defined as

$$
D_{B}:=\sum_{N \geqslant j>i \geqslant 1} H_{j, i}+\sum_{i=1}^{N} D_{i} .
$$

Where it does not cause confusion, we shorten $D_{B}$ to simply $D$. 


\section{Perfect KR crystals and Demazure crystals}

We now state a precise relationship between KR crystals and Demazure crystals (see Theorem 6.1). This was proven by Fourier, Schilling, and Shimozono [12], under a few additional assumptions on the KR crystals since at the time the existence and combinatorial models for KR crystals did not yet exist. Here we point out that most of the assumptions in [12] follow from the later results of $[49,50,10,11]$ showing that the relevant KR crystals exist, have certain symmetries related to Dynkin diagram automorphisms, and are perfect. In the special cases of type $A_{2 n}^{(2)}$ and the exceptional nodes in type $D_{n}^{(1)}$, we give separate proofs as the assumptions from [12] do not follow directly from the above papers or need to be slightly modified.

Theorem 6.1. Let $B=B^{r_{N}, \ell c_{r_{N}}} \otimes \cdots \otimes B^{r_{1}, \ell c_{r_{1}}}$ be a level- $\ell$ composite KR crystal of nonexceptional type. Define $\lambda=-\left(c_{r_{1}} \omega_{r_{1}^{*}}+\cdots+c_{r_{N}} \omega_{r_{N}^{*}}\right)$, where $r^{*}$ is defined by $\omega_{r^{*}}=-w_{0}\left(\omega_{r}\right)$ with $w_{0}$ the longest element of $\bar{W}$, and write $t_{\lambda} \in T(\widetilde{M}) \subset \widetilde{W}$ as $t_{\lambda}=v \tau$. Then there is a unique isomorphism of affine crystals

$$
j: B\left(\ell \Lambda_{\tau(0)}\right) \rightarrow B \otimes B\left(\ell \Lambda_{0}\right)
$$

This satisfies

$$
j\left(u_{\ell \Lambda_{\tau(0)}}\right)=u_{B} \otimes u_{\ell \Lambda_{0}}
$$

where $u_{B}$ is the distinguished element from Definition 5.2, and

$$
j\left(B_{v}\left(\ell \Lambda_{\tau(0)}\right)\right)=B \otimes u_{\ell \Lambda_{0}}
$$

where $B_{v}\left(\ell \Lambda_{\tau(0)}\right)$ is the Demazure crystal corresponding to the translation $t_{\lambda}$ as defined in Section 2.9.

We delay the proof of Theorem 6.1 until the end of this section.

Remark 6.2. For non-exceptional types, we have $r^{*}=r$ except for type $A_{n}^{(1)}$ where $r^{*}=n+1-r$, and $D_{n}^{(1)}$ for $n$ odd where $n^{*}=n-1$ and $(n-1)^{*}=n$.

Lemma 6.3. Assume $\mathfrak{g}$ is of non-exceptional type, and let $B^{r, \ell c_{r}}$ be a level- $\ell K R$ crystal. Then:

(i) There is a unique element $u \in B^{r, l_{r}}$ such that

$$
\varepsilon(u)=\ell \Lambda_{0} \quad \text { and } \quad \varphi(u)=\ell \Lambda_{\tau(0)},
$$

where $t_{-c_{r} \omega_{r^{*}}}=v \tau$ with $v \in W$ and $\tau \in \Sigma$.

(ii) Let $\varsigma$ be the Dynkin diagram automorphism defined by

- For type $A_{n}^{(1)}, i \mapsto i+1(\bmod n+1)$.

- For types $B_{n}^{(1)}, D_{n}^{(1)}, A_{2 n-1}^{(2)}$, exchange nodes 0 and 1. 
- For types $C_{n}^{(1)}$ and $D_{n+1}^{(2)}, i \mapsto n-i$ for all $i \in I$.

In all cases other than type $A_{2 n}^{(2)}$ and $B^{n-1, s}$ and $B^{n, s}$ for type $D_{n}^{(1)}$, there is a unique involution of $B^{r, \ell c_{r}}$, also denoted $\varsigma$, such that for all $b \in B^{r, \ell c_{r}}$ and $i \in I$,

$$
f_{i}(b)=\varsigma^{-1} \circ f_{\varsigma(i)} \circ \varsigma(b) .
$$

Remark 6.4. Lemma 6.3 was stated as [12, Assumption 1] (with the misprint $t_{-c_{r} \omega_{r}}$ instead of $t_{-c_{r} \omega_{r^{*}}}$ in (i)). This Assumption also included the requirement that $B^{r, \ell c_{r}}$ is regular. Since we now know that $B^{r, \ell c_{r}}$ is the crystal of a $\operatorname{KR}$ module by $[49,50,10]$, this is immediate, and so we do not include it in Lemma 6.3. The statement in [12] also included an additional assumption for type $A_{2 n}^{(2)}$, which we omit as we deal with type $A_{2 n}^{(2)}$ separately.

Proof of Lemma 6.3. By perfectness of $B^{r, \ell c_{r}}$ for non-exceptional types [11], there exists a unique element $u \in B^{r, \ell c_{r}}$ such that $\varepsilon(u)=\ell \Lambda_{0}$. These elements are listed for all non-exceptional types in Figure 4. It is easy to check explicitly that these also satisfy $\varphi(u)=\ell \Lambda_{\tau(0)}$, where $\tau$ is as listed. Furthermore, one can easily check that all these $\tau$ satisfy the remaining conditions of (i).

The combinatorial model for $B^{r, s}$ of type $A_{n}^{(1)}$ uses the promotion operator which corresponds to the Dynkin diagram automorphism mapping $i \mapsto i+1(\bmod n+1)$. Similarly, the KR crystals in case (AUT) are constructed using the automorphism $\varsigma$ (see Section 4.3).

By Theorem 4.7 the KR crystal $B^{n, 2 \ell}$ of type $B_{n}^{(1)}$ can be constructed as a virtual crystal inside $\hat{B}^{n, 2 \ell}$ of type $A_{2 n-1}^{(2)}$ with $e_{0}=\hat{e}_{0}^{2}$ and $e_{1}=\hat{e}_{1}^{2}$. The virtual crystal is constructed using the analogue of the Dynkin automorphism $\hat{\varsigma}$ exchanging 0 and 1 , so that $e_{0}=\hat{e}_{0}^{2}=\left(\hat{\varsigma} \hat{e}_{1} \hat{\varsigma}\right)\left(\hat{\varsigma} \hat{e}_{1} \hat{\varsigma}\right)=\hat{\varsigma} \hat{e}_{1}^{2} \hat{\varsigma}=\hat{\varsigma} e_{1} \hat{\varsigma}$. Furthermore, by [10, Lemma 3.5] the image of $B^{n, 2 \ell}$ in the ambient crystals $\hat{B}^{n, 2 \ell}$ is closed under $\hat{\varsigma}$. Hence on $B^{n, 2 \ell}$ of type $B_{n}^{(1)}$ there also exists an isomorphism interchanging nodes 0 and 1 , induced by $\hat{\varsigma}$.

It was shown in [10, Theorem 7.1] that $B^{r, s}$ of types $C_{n}^{(1)}$ and $D_{n+1}^{(2)}$ admit a twisted isomorphism $\varsigma$ corresponding to the Dynkin automorphism mapping $i \mapsto n-i$ for $i \in I$. This shows (ii).

For $B$ a crystal of affine type and $\tau \in \operatorname{Aut}(\Gamma)$, where $\Gamma$ is the affine Dynkin diagram, let $B^{\tau}$ be the crystal with the same underlying set as $B$, but where $e_{i}^{\tau}=\tau \circ e_{i} \circ \tau^{-1}$ and $f_{i}^{\tau}=\tau \circ f_{i} \circ \tau^{-1}$.

Lemma 6.5. Let $\Gamma$ be a non-exceptional affine Dynkin diagram and $\tau \in \Sigma$. Then $\left(B^{r, s}\right)^{\tau} \cong B^{r, s}$ for all $r \in I \backslash\{0\}$ and $s \geqslant 1$.

Proof. Fix $\tau \in \Sigma$. By Lemma 2.8, there exists $w_{\tau} \in \bar{W}$ such that

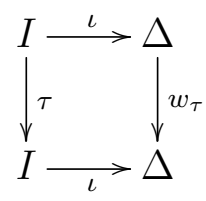




\begin{tabular}{|l|l|c|l|l|}
\hline Type & $u \in B^{r, \ell c_{r}}$ & $r$ & $c_{r}$ & $\tau$ \\
\hline \hline$A_{n}^{(1)}$ & $u\left(\ell \Lambda_{r}\right)$ & $1 \leqslant r \leqslant n$ & 1 & $\mathrm{pr}^{r}$ \\
\hline$B_{n}^{(1)}$ & $u(\emptyset)$ & $1 \leqslant r<n, r$ even & 1 & $\mathrm{id}$ \\
& $u\left(\ell \Lambda_{1}\right)$ & $1 \leqslant r<n, r$ odd & 1 & $\varsigma_{0,1}$ \\
& $u(\emptyset)$ & $r=n$ even & 2 & $\mathrm{id}$ \\
& $u\left(\ell \Lambda_{1}\right)$ & $r=n$ odd & 2 & $\varsigma_{0,1}$ \\
\hline$C_{n}^{(1)}$ & $u(\emptyset)$ & $1 \leqslant r<n$ & 2 & $\mathrm{id}$ \\
& $u\left(\ell \Lambda_{n}\right)$ & $r=n$ & 1 & $\varsigma_{\leftrightarrow}$ \\
\hline$D_{n}^{(1)}$ & $u(\emptyset)$ & $1 \leqslant r \leqslant n-2, r$ even & 1 & $\mathrm{id}$ \\
& $u\left(\ell \Lambda_{1}\right)$ & $1 \leqslant r \leqslant n-2, r$ odd & 1 & $\varsigma_{0,1} \varsigma_{n-1, n}$ \\
& $u\left(\ell \Lambda_{n-1}\right)$ & $r=n-1$ & 1 & $\varsigma_{\leftrightarrow} \varsigma_{0,1} \varsigma_{n-1, n}^{n+1}$ \\
& $u\left(\ell \Lambda_{n}\right)$ & $r=n$ & 1 & $\varsigma_{\leftrightarrow} \varsigma_{n-1, n}^{n}$ \\
\hline$A_{2 n-1}^{(2)}$ & $u(\emptyset)$ & $1 \leqslant r \leqslant n, r$ even & 1 & $\mathrm{id}$ \\
& $u\left(\ell \Lambda_{1}\right)$ & $1 \leqslant r \leqslant n, r$ odd & 1 & $\varsigma_{0,1}$ \\
\hline$D_{n+1}^{(2)}$ & $u(\emptyset)$ & $1 \leqslant r<n$ & 1 & $\mathrm{id}$ \\
& $u\left(\ell \Lambda_{n}\right)$ & $r=n$ & 1 & $\varsigma_{\leftrightarrow}$ \\
\hline$A_{2 n}^{(2)}$ & $u(\emptyset)$ & $1 \leqslant r \leqslant n$ & 1 & $\mathrm{id}$ \\
\hline
\end{tabular}

Figure 4: The elements $u$ and related data. Here $u(\lambda)$ is the highest weight vector in the classical component $B(\lambda)$, pr is the map $i \mapsto i+1(\bmod n+1)$, and $\varsigma_{0,1}\left(\operatorname{resp} . \varsigma_{n-1, n}\right)$ is the map that interchanges 0 and 1 (resp. $n-1$ and $n$ ) and fixes all other $i$. The map $\varsigma_{\leftrightarrow}$ is $i \mapsto n-i$. In the expression for $\tau$ the maps act on the left, so that e.g. $\varsigma_{\leftrightarrow} \varsigma_{0,1}(1)=\varsigma_{\leftrightarrow}(0)=n$.

commutes, where $\iota$ is the map that takes $i$ to $\alpha_{i}$ for all $i \neq 0$, and 0 to $-\theta$ (where $\left.\theta=\delta-\alpha_{0}\right)$. Thus the character of $\left(B^{r, s}\right)^{\tau}$ as a $\{1,2, \ldots, n\}$-crystal is in the Weyl group orbit of the character of $B^{r, s}$ as a $\{1,2, \ldots, n\}$-crystal, and hence by Weyl group invariance these are equal. By the classification of classical crystals, it follows that $\left(B^{r, s}\right)^{\tau} \cong B^{r, s}$ as a $\{1,2, \ldots, n\}$-crystal. Thus the lemma follows by Lemma 2.6.

Lemma 6.6. Theorem 6.1 holds in type $D_{n}^{(1)}$.

Proof. For $B=B^{r, \ell}$ with $1 \leqslant r \leqslant n-2$, Lemma 6.3 shows that [12, Assumption 1$]$ holds, and so Theorem 6.1 holds by [12, Theorem 4.4].

Now consider $B^{n, \ell}$, noting that $c_{n}=1$. We need the following notation:

- As in Theorem 6.1, $n^{*}$ is defined by $\omega_{n^{*}}=-w_{0}\left(\omega_{n}\right)$, so that $\left(w_{0}^{n^{*}}\right)^{-1}=w_{0}^{n}$.

- $w_{0}^{n} \in \bar{W}$ is of minimal length such that $w_{0}^{n}\left(\omega_{n}\right)=w_{0}\left(\omega_{n}\right)$,

- $w_{0}^{n^{*}}$ is of minimal length such that $w_{0}^{n^{*}}\left(\omega_{n^{*}}\right)=w_{0}\left(\omega_{n^{*}}\right)$,

- $\tau_{n}$ and $\tau_{n^{*}}$ are the $\tau$ from Table 4 for $r=n, n^{*}$ respectively.

As in [12, Equation (2.10)] (but noting that due to differing conventions their $\tau_{n^{*}}$ is our $\left.\tau_{n}\right)$,

$$
t_{-\omega_{n^{*}}}=\left(w_{0}^{n^{*}}\right)^{-1} \tau_{n}=w_{0}^{n} \tau_{n}
$$


Since $w_{0}^{n}\left(\ell \Lambda_{n}\right)=w_{0}\left(\ell \Lambda_{n}\right)$, we see that

$$
B_{w_{0}^{n}}\left(\ell \Lambda_{n}\right)=B_{w_{0}}\left(\ell \Lambda_{n}\right)=\left\{f_{i_{N}}^{m_{N}} \cdots f_{i_{1}}^{m_{1}} u_{\ell \Lambda_{n}} \mid m_{1}, \ldots, m_{N} \geqslant 0\right\}
$$

where $s_{i_{1}} \cdots s_{i_{N}}$ is a reduced word for $w_{0}$ and the last equality follows by [27, Proposition 3.2.3] (or see Section 2.9 above).

As in Section $4.1, B^{n, \ell}$ is isomorphic to $B\left(\ell \Lambda_{n}\right)$ as a classical crystal. The element $u$ from Figure 4 is the highest weight element $u\left(\ell \Lambda_{n}\right)$ of weight $\ell \Lambda_{n}$. It satisfies $\varepsilon\left(u\left(\ell \Lambda_{n}\right)\right)=$ $\ell \Lambda_{0}$ and $\varphi\left(u\left(\ell \Lambda_{n}\right)\right)=\ell \Lambda_{n}$. Furthermore $B^{n, \ell}$ is perfect of level $\ell$ so, as in [25], there is a unique isomorphism

$$
\begin{aligned}
j: B\left(\ell \Lambda_{n}\right) & \rightarrow B^{n, \ell} \otimes B\left(\ell \Lambda_{0}\right) \\
u_{\ell \Lambda_{n}} & \mapsto u\left(\ell \Lambda_{n}\right) \otimes u_{\ell \Lambda_{0}} .
\end{aligned}
$$

Since $\varepsilon_{i}\left(u_{\ell \Lambda_{0}}\right)=\varphi_{i}\left(u_{\ell \Lambda_{0}}\right)=0$ for all $i \neq 0,(6.4)$ and (6.5) imply

$$
j\left(B_{w_{0}^{n}}\left(\ell \Lambda_{n}\right)\right)=B^{n, \ell} \otimes u_{\ell \Lambda_{0}} .
$$

Since $\tau_{n}(0)=n$, we have proven Theorem 6.1 for $B=B^{n, s}$. A similar argument shows that Theorem 6.1 holds for $B=B^{n-1, s}$.

By Lemma 6.5, each automorphism $\tau \in \Sigma$ induces a bijection of $B^{r, \ell}$ to itself that sends $i$ arrows to $\tau(i)$ arrows. Thus, as in [12, Theorem 4.7], a straightforward induction argument shows that Theorem 6.1 holds for all composite level- $\ell$ KR crystals.

Lemma 6.7. Theorem 6.1 holds in type $A_{2 n}^{(2)}$.

Proof. In [12] a proof of Theorem 6.1 was given, but not with the concrete combinatorial model given in Theorem 4.9. The only place, where the concrete combinatorial model for type $A_{2 n}^{(2)}$ is used in [12] is in the proof of [12, Lemma 4.3]. Thus we must verify that this lemma holds with the explicit realization of KR crystals for this type from [10] or equivalently Theorem 4.9. Let $S: B^{r, s} \rightarrow \hat{B}_{C_{n}^{(1)}}^{r, 2 s}$ be the unique injective map from the combinatorial KR crystal of type $A_{2 n}^{(2)}$ to the KR crystal of type $C_{n}^{(1)}$ such that

$$
S\left(e_{i} b\right)=\hat{e}_{i}^{m_{i}} S(b) \quad \text { and } \quad S\left(f_{i} b\right)=\hat{f}_{i}^{m_{i}} S(b) \quad \text { for all } i \in I \text { and } b \in B^{r, s},
$$

where $m=\left(m_{0}, \ldots, m_{n}\right)=(1,2, \ldots, 2)$. Using the explicit realization of $\hat{B}_{C_{n}^{(1)}}^{r, 2 s}$ it is not too hard to check that for the classically highest weight element $u\left(s \omega_{k}\right) \in B\left(s \omega_{k}\right) \subset B^{r, s}$ for $k<r$ and $y:=S_{1} \cdots S_{k}\left(u\left(s \omega_{k}\right)\right.$ ) (where $S_{i}$ is $f_{i}$ raised to the maximal power), we have $S\left(f_{0}^{s} y\right)=S\left(u\left(s \omega_{k+1}\right)\right)$. Thus [12, Lemma 4.3] holds. Furthermore, in this case $\tau$ is always the identity. Thus Theorem 6.1 holds by [12, Theorem 4.7].

Proof of Theorem 6.1. If $X=D_{n}^{(1)}$ or $A_{2 n}^{(2)}$, the Theorem holds by Lemmas 6.6 and 6.7. In all other cases, Lemma 6.3 shows that [12, Assumption 1] holds. Furthermore, in all these cases $\Sigma$ is generated by the diagram automorphism $\varsigma$ from Lemma 6.3. Thus the theorem holds by [12, Theorem 4.7]. 


\section{The affine grading via the energy function}

In this section, we show that for $B=B^{r_{N}, \ell c_{r_{N}}} \otimes \cdots \otimes B^{r_{1}, \ell c_{r_{1}}}$ a composite level- $\ell \mathrm{KR}$ crystal the map $j$ from Theorem 6.1 intertwines the $D$ function from Section 5.2 with the affine degree deg given in Definition 7.1 below up to a shift. This allows us to show that $j$ intertwines $E^{\text {int }}$ with deg exactly, and in particular $E^{\text {int }}$ agrees with $D$ up to a shift.

Definition 7.1. For any Demazure crystal $B_{w}(\Lambda)$, where $w \in W$ and $\Lambda$ is a dominant integral weight, let

$$
\operatorname{deg}: B_{w}(\Lambda) \rightarrow \mathbb{Z}_{\geqslant 0}
$$

be the affine degree map defined by $\operatorname{deg}\left(u_{\Lambda}\right)=0$ and each $f_{i}$ has degree $\delta_{i, 0}$.

We begin by two preliminary lemmas, first for a single KR crystal and then tensor products of KR crystals.

Lemma 7.2. Fix a KR crystal $B^{r, s}$ of type $\mathfrak{g}$. Then $D\left(e_{0}(b)\right) \geqslant D(b)-1$ for all $b \in B^{r, s}$. Furthermore, if $\varepsilon_{0}(b)>\left\lceil s / c_{r}\right\rceil$, then we have $D\left(e_{0}(b)\right)=D(b)-1$.

Proof.

- Case (IRR): In this case $\diamond=\emptyset$, so that by Definition 5.4 the $D$ function is the constant function 0 which satisfies $D\left(e_{0}(b)\right) \geqslant D(b)-1$ for all $b \in B^{r, s}$. By Lemma 4.1 we always have $\varepsilon_{0}(b) \leqslant s / c_{r}$ since $c_{r}=1$.

- Case (AUT): In this case $\diamond$ is a vertical domino. The statements follow immediately from Lemma 4.4 since again $c_{r}=1$ in all cases.

- For $B^{r, s}$ of type $C_{n}^{(1)}$ with $r<n$, we have $c_{r}=2$ and $\diamond$ is a horizontal domino. The result follows from Lemma 4.6.

- For $B^{n, s}$ of type $B_{n}^{(1)}$, we have $c_{r}=2$ and $\diamond$ is a vertical domino. The result follows from Lemma 4.8 .

- For $B^{r, s}$ of types $A_{2 n}^{(2)}$ with $r \leqslant n$ and $D_{n+1}^{(2)}$ with $r \leqslant n-1$, we have $c_{r}=1$ and $\diamond$ is a single box. The result follows from Lemma 4.10.

Lemma 7.3. Let $B=B^{r_{N}, s_{N}} \otimes \cdots \otimes B^{r_{1}, s_{1}}$ be a tensor product of $K R$ crystals and fix an integer $\ell$ such that $\ell \geqslant\left\lceil s_{k} / c_{k}\right\rceil$ for all $1 \leqslant k \leqslant N$. If $e_{0}(b) \neq 0$ then $D\left(e_{0}(b)\right) \geqslant D(b)-1$, and if $\varepsilon_{0}(b)>\ell$ then this is an equality.

Proof. Write $b=b_{N} \otimes \cdots \otimes b_{1}$. For some $N \geqslant k \geqslant 1$,

$$
e_{0}(b)=b_{N} \otimes \cdots \otimes e_{0}\left(b_{k}\right) \otimes \cdots \otimes b_{1} .
$$

Let

$$
b_{N}^{\prime} \otimes \cdots \otimes b_{1}^{\prime}=\sigma_{1} \cdots \sigma_{k-1}(b)
$$


Since each $\sigma_{j}$ commutes with the action of $e_{0}$,

$$
e_{0}\left(b_{N}^{\prime} \otimes \cdots \otimes b_{1}^{\prime}\right)=b_{N}^{\prime} \otimes \cdots \otimes e_{0}\left(b_{c}^{\prime}\right) \otimes \cdots \otimes b_{1}^{\prime}
$$

for some $k \geqslant c \geqslant 1$. For each $1 \leqslant x \leqslant N$, let

$$
D^{[x]}=\sum_{1 \leqslant y<x} H_{x, y}+D_{x}
$$

where $H_{x, y}$ and $D_{x}$ are as in Definition 5.5, so that $D=\sum_{1 \leqslant x \leqslant N} D^{[x]}$. Let $\Delta(b)=$ $D(b)-D\left(e_{0}(b)\right)$ and $\Delta^{[x]}(b)=D^{[x]}(b)-D^{[x]}\left(e_{0}(b)\right)$. We find each $\Delta^{[x]}(b)$, considering three cases.

Case $1, k>x$ : Here $\Delta^{[x]}(b)=0$, as all terms in $D^{[x]}(b)$ and $D^{[x]}\left(e_{0}(b)\right)$ agree.

Case 2, $x=k$ : If $c \neq 1$, then, by Definitions 5.4 and 5.5, $D_{x}(b)=D_{x}\left(e_{0}(b)\right)$ and by $(5.1), H_{x, c-1}\left(e_{0}(b)\right)=H_{x, c-1}(b)-1$. For all other $y<x$ it is clear that $H_{x, y}(b)=$ $H_{x, y}\left(e_{0}(b)\right)$. Hence $\Delta^{[x]}(b)=1$.

Otherwise $c=1$ and

$$
\Delta^{[x]}(b)=D^{[x]}(b)-D^{[x]}\left(e_{0}(b)\right)=D_{B^{r_{1}, s_{1}}}\left(b_{1}^{\prime}\right)-D_{B^{r_{1}, s_{1}}}\left(e_{0}\left(b_{1}^{\prime}\right)\right) .
$$

In this case $\varepsilon\left(b_{1}^{\prime}\right)=\varepsilon(b)$, so by Lemma $7.2, \Delta^{[x]}(b) \leqslant 1$, with equality if $\varepsilon_{0}(b)>\ell \geqslant s_{r_{1}} / c_{r_{1}}$.

Case 3, $x>k$ : It is clear that $H_{x, y}(b)=H_{x, y}\left(e_{0}(b)\right)$ for $x>y>k$. Let

$$
d=d_{N} \otimes \cdots \otimes d_{1}:=\sigma_{k} \cdots \sigma_{x-1}(b)
$$

Since each $\sigma_{j}$ commutes with the action of $e_{0}$, one of the following must hold:

(a) $e_{0}\left(d_{N} \otimes \cdots \otimes d_{1}\right)=d_{N} \otimes \cdots \otimes e_{0}\left(d_{k+1}\right) \otimes d_{k} \otimes \cdots \otimes d_{1}$,

(b) $e_{0}\left(d_{N} \otimes \cdots \otimes d_{1}\right)=d_{N} \otimes \cdots \otimes d_{k+1} \otimes e_{0}\left(d_{k}\right) \otimes \cdots \otimes d_{1}$.

If (a) holds, then for $y<k$ we have $H_{x, y}(b)=H_{x, y}\left(e_{0}(b)\right)$, since $H_{x, y}$ is calculated on exactly the same tensor product on each side. By (5.1) we have $H_{x, k}(b)=H_{x, k}\left(e_{0}(b)\right)$ and by Definition $5.4 D_{x}(b)=D_{x}\left(e_{0}(b)\right)$. Hence $\Delta^{[x]}(b)=0$.

If (b) holds, then, by (5.1), $H_{x, k}\left(e_{0}(b)\right)=H_{x, k}(b)+1$, and for all $k<y<x$, $H_{x, y}\left(e_{0}(b)\right)=H_{x, y}(b)$. Furthermore, by Definition $5.5 D_{x}(b)=D_{k}(d)$ and $D_{x}\left(e_{0} b\right)=$ $D_{k}\left(e_{0} d\right)$. Comparing terms and noticing that $H_{x, r}(b)=H_{k, r}(d)$ and $H_{x, r}\left(e_{0} b\right)=H_{k, r}\left(e_{0} d\right)$ for all $r<x$ shows

$$
\Delta^{[x]}(b)=\Delta^{[k]}(d)-1,
$$

so it follows by the argument in Case 2 that $\Delta^{[x]}(b) \leqslant 0$, with equality if $\varepsilon_{0}(b)=\varepsilon_{0}(d)>\ell$.

The result now follows by adding all the $\Delta^{[x]}(b)$.

Theorem 7.4. With the same assumptions and notation as in Theorem 6.1, let $\tilde{j}$ : $B_{v}\left(\ell \Lambda_{\tau(0)}\right) \rightarrow B$ be the restriction of the map $j$ to $B_{v}\left(\ell \Lambda_{\tau(0)}\right)$, where $B \otimes u_{\ell} \Lambda_{0}$ is identified with just $B$. Then for all $b \in B_{v}\left(\ell \Lambda_{\tau(0)}\right)$ we have $\operatorname{deg}(b)=D(\tilde{j}(b))-D\left(u_{B}\right)$. 
Proof. Since $B_{v}\left(\ell \Lambda_{\tau(0)}\right)$ is connected with the highest weight element $u_{\ell \Lambda_{\tau(0)}}, \tilde{j}\left(u_{B}\right)=$ $u_{\ell \Lambda_{\tau(0)}}$, and $\operatorname{deg}\left(u_{\ell \Lambda_{\tau(0)}}\right)=0$, it suffices to show that $D(\tilde{j}(b))=D\left(\tilde{j}\left(e_{0}(b)\right)\right)-1$ for each $b \in B_{v}\left(\ell \Lambda_{\tau(0)}\right)$ such that $e_{0}(b) \neq 0$. So, choose such a $b \in B_{v}\left(\ell \Lambda_{\tau(0)}\right)$. Since $e_{0}$ acts nontrivially on $\tilde{j}(b) \otimes u_{\ell \Lambda_{0}}$, we have $\varepsilon_{0}(\tilde{j}(b))>\ell$, and the result follows by Lemma 7.3.

Theorem 7.5. For any composite level- $\ell K R$ crystal $B=B^{r_{N}, \ell c_{r_{N}}} \otimes \cdots \otimes B^{r_{1}, \ell c_{r_{1}}}$ we have $E^{\text {int }}=D-D\left(u_{B}\right)$.

Proof. By the definition of $E^{\text {int }}$ (see Section 5.1), for any $b$ there exists a string of $f_{i}$ involving exactly $E^{\text {int }}(b)$ factors $f_{0}$ taking $u_{B}$ to $b$. By Lemma 7.3, applying $f_{0}$ increases the value of $D$ by at most 1 , and so it follows that $E^{\text {int }}(b) \geqslant D(b)-D\left(u_{B}\right)$. It follows from Theorem 7.4 and the fact that the Demazure crystal $B_{v}\left(\ell \Lambda_{\tau(0)}\right)$ is connected that $E^{\text {int }}(b) \leqslant D(b)-D\left(u_{B}\right)$.

Corollary 7.6. The map $j$ intertwines $E^{\text {int }}$ and the basic grading deg.

Proof. This is immediate from Theorems 7.4 and 7.5 .

Remark 7.7. Let $B$ be a composite level- $\ell$ KR crystal. If $\varepsilon_{0}(b)>\ell$, call the corresponding 0 arrow from $b^{\prime}=e_{0}(b)$ to $b$ in $B$ a Demazure arrow. We have shown that for such an arrow $D(b)=D\left(b^{\prime}\right)+1$. Furthermore, we have shown that $u_{B}$ is the unique source in the subgraph of $B$ consisting only of classical arrows (i.e. arrows colored $i$ for some $i \neq 0$ ) and Demazure arrows. This could be used to give an algorithm for calculating the energy function: given $b$, move backwards along arrows until one reach $u_{B}$, only using Demazure 0-arrows. Then $D(b)-D\left(u_{B}\right)$ is the number of 0 arrows in this path.

\section{Generalizations to other tensor products of KR crystals}

Notice that Lemma 7.3 holds for more general tensor products of KR crystals, not just tensor products of level- $\ell$ perfect KR crystals. We now show how Theorem 7.4 and Corollary 7.6 can be generalized as well.

For this section, fix $\mathfrak{g}$ of non-exceptional affine type, $\ell>0$, and a tensor product $B=B^{r_{N}, s_{N}} \otimes \cdots \otimes B^{r_{1}, s_{1}}$ of KR crystals, such that $\ell \geqslant\left\lceil s_{k} / c_{k}\right\rceil$ for all $1 \leqslant k \leqslant N$. We call such a crystal a composite $K R$ crystal of level bounded by $\ell$.

The proof of the following proposition is similar to [25, Proof of Theorem 4.4.1].

Proposition 8.1. For $B$ a composite KR crystal of level bounded by $\ell$,

$$
B \otimes B\left(\ell \Lambda_{0}\right) \cong \bigoplus_{\Lambda^{\prime}} B\left(\Lambda^{\prime}\right)
$$

where the sum is over a finite collection of (not necessarily distinct) $\Lambda^{\prime} \in P_{\ell}^{+}$. 
Proof. Fix $b \otimes c \in B \otimes B\left(\ell \Lambda_{0}\right)$. If $c \neq u_{\ell \Lambda_{0}}$, then for some $i \in I$ we have $e_{i}(c) \neq 0$. This implies that, for some $k \geqslant 1$,

$$
e_{i}^{k}(b \otimes c)=e_{i}^{k-1}(b) \otimes e_{i}(c) \neq 0 .
$$

In this way, one can apply raising operators to $b \otimes c$ to obtain an element $b^{\prime} \otimes u_{\ell \Lambda_{0}}$.

Set $M=\operatorname{dim}(B)$, and assume that, for some choice of $e_{i_{1}}, \ldots, e_{i_{M}}$, and some $b \in B$, $e_{i_{M}} \cdots e_{i_{1}}\left(b \otimes u_{\ell \Lambda_{0}}\right) \neq 0$. Then some element $b^{\prime} \otimes u_{\ell \Lambda_{0}}$ must appear twice. Thus for some $1 \leqslant r \leqslant s \leqslant M$, we have $e_{i_{s}} \cdots e_{i_{r}}\left(b^{\prime} \otimes u_{\ell \Lambda_{0}}\right)=b^{\prime} \otimes u_{\ell \Lambda_{0}}$. By weight considerations, at least one $e_{0}$ must appear in this sequence. Furthermore, whenever $e_{i_{k}}=e_{0}$, we must have $\varepsilon_{0}\left(e_{i_{k-1}} \cdots e_{i_{r}}\left(b^{\prime}\right)\right)>\ell$. Thus Lemma 7.3 implies that $D\left(b^{\prime}\right)<D\left(b^{\prime}\right)$, which is a contradiction. Hence the left hand side does decompose as a finite direct sum of $B\left(\Lambda^{\prime}\right)$ for $\Lambda^{\prime} \in P^{+}$. It follows by weight considerations that all $\Lambda^{\prime}$ are in $P_{\ell}^{+}$.

Definition 8.2. For each $b \in B$, let $u_{b}^{\ell \Lambda_{0}}$ be the unique element of $B$ such that $u_{b}^{\ell \Lambda_{0}} \otimes u_{\ell \Lambda_{0}}$ is the highest weight in the component from Proposition 8.1 containing $b \otimes u_{\ell \Lambda_{0}}$.

Define the function deg on a direct sum of highest weight crystals to be the basic grading on each component, with all highest weight elements placed in degree 0.

Corollary 8.3. Choose an isomorphism $m: B \otimes B\left(\ell \Lambda_{0}\right) \cong \bigoplus_{\Lambda^{\prime}} B\left(\Lambda^{\prime}\right)$. Then for all $b \in B$, we have $D(b)-D\left(u_{b}^{\ell \Lambda_{0}}\right)=\operatorname{deg}\left(m\left(b \otimes u_{\ell \Lambda_{0}}\right)\right)$.

Proof. This follows from Lemma 7.3, just as in the proof of Theorem 7.4.

The following should be interpreted as a generalization of Corollary 7.6.

Corollary 8.4. The minimal number of $e_{0}$ in a string of $e_{i}$ taking $b$ to $u_{b}^{\ell \Lambda_{0}}$ is $D(b)-$ $D\left(u_{b}^{\ell \Lambda_{0}}\right)$.

Proof. This follows as in the proof of Corollary 7.6, using the fact that, for any $b \in B$, $b \otimes u_{\ell \Lambda_{0}}$ is connected to $u_{b}^{\ell \Lambda_{0}} \otimes u_{\ell \Lambda_{0}}$ in the affine highest weight crystal.

Remark 8.5. The statements in this section do not give a relationship with Demazure crystals. However, Naoi [46, Proposition after Theorem B, Proposition 7.6] and [47] has recently proven that $B \otimes u_{\Lambda}$ is isomorphic to a disjoint union of Demazure crystals inside the various irreducible components of $B \otimes B(\Lambda)$, where $\Lambda$ is an arbitrary dominant weight of level $\ell \geqslant 1$ and $B$ is a tensor product of perfect KR crystals each of which has level at most $\ell$. It would be interesting to determine whether or not this continues to hold for $B$ nonperfect.

\section{Applications}

In this section we discuss how the relation between the affine grading in the Demazure crystal and the energy function can be used to derive a formula for the Demazure character using the energy function, as well as showing how they are related to nonsymmetric Macdonald polynomials and Whittaker functions. 


\subsection{Demazure characters}

Since the character of a Demazure module $V_{w}(\lambda)$ can be expressed in terms of the Demazure crystal by (2.9), we have the following immediate corollary of Theorem 6.1 .

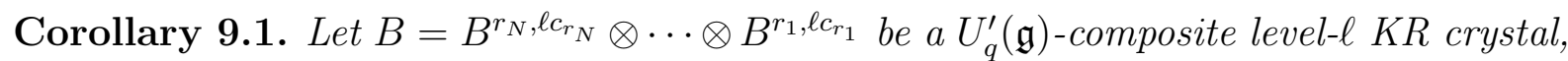
$\lambda=-\left(c_{r_{1}} \omega_{r_{1}^{*}}+\cdots+c_{r_{N}} \omega_{r_{N}^{*}}\right)$, and $t_{\lambda}=v \tau$ as in Theorem 6.1. Then

$$
\operatorname{ch} V_{v}\left(\ell \Lambda_{\tau(0)}\right)=e^{\ell \Lambda_{0}} \sum_{b \in B} e^{\mathrm{wt}(b)-\delta E^{\mathrm{int}}(b)}=e^{\ell \Lambda_{0}} \sum_{b \in B} e^{\mathrm{wt} \mathrm{wff}^{(b)}}
$$

where wt ${ }^{\text {aff }}(b)=\mathrm{wt}(b)-\delta E^{\mathrm{int}}(b)$ and wt $(b)$ is the $U_{q}^{\prime}(\mathfrak{g})$-weight of $b$.

Proof. Recall that by Theorem 6.1 we have the isomorphism

$$
j\left(B_{v}\left(\ell \Lambda_{\tau(0)}\right)\right)=B \otimes u_{\ell \Lambda_{0}} .
$$

Combining this with (2.9), we obtain the result.

As in e.g. [16, 17], the one-dimensional configuration sums are defined as

$$
X(\mu ; B)=\sum_{\substack{b \in B, \mathrm{wt}(b)=\mu \\ e_{i}(b)=\emptyset \text { for } i \in I \backslash\{0\}}} q^{-D(b)},
$$

where $B=B^{r_{N}, s_{N}} \otimes \cdots \otimes B^{r_{1}, s_{1}}$. By Theorem 7.5 we can rewrite Corollary 9.1 as follows (compare also with [54, Theorem 1.2]).

Corollary 9.2. With the same assumptions and notation as in Corollary 9.1 and setting $q=e^{\delta}$ we have

$$
\operatorname{ch} V_{v}\left(\ell \Lambda_{\tau(0)}\right)=e^{\ell \Lambda_{0}} q^{D\left(u_{B}\right)} \sum_{\mu} X(\mu ; B) \operatorname{ch}(V(\mu)),
$$

where $V(\mu)$ is the module of highest weight $\mu$ for the underlying finite type algebra.

Example 9.3. Let us illustrate various quantities used in this section. Consider $B=$ $\left(B^{1,1}\right)^{\otimes 3}$ of type $A_{2}^{(1)}$. This has $u_{B}=3 \otimes 2 \otimes 1$. Take $b=2 \otimes 3 \otimes 1$. Then one can calculate $D(b)=-2$ and $D\left(u_{B}\right)=-3$, so that $E^{\text {int }}(b)=D(b)-D\left(u_{B}\right)=1$. Also

$$
\operatorname{wt}(b)=\left(\Lambda_{2}-\Lambda_{1}\right)+\left(\Lambda_{0}-\Lambda_{2}\right)+\left(\Lambda_{1}-\Lambda_{0}\right)=0,
$$

so that wt ${ }^{\text {aff }}(b)=-\delta$. 


\subsection{Nonsymmetric Macdonald polynomials}

Fix $\mathfrak{g}$ of affine type. Let $\widetilde{P} \subset P$ be the sublattice of level 0 weights. Recall that $\widetilde{P}$ is naturally contained in $\bar{P}+\mathbb{Z} \delta$, where $\delta$ is the null root and we identify $\bar{P} \otimes_{\mathbb{Z}} \mathbb{R}$ with a subspace of $P \otimes_{\mathbb{Z}} \mathbb{R}$ by identifying the finite type simple roots with their corresponding affine simple roots (and this containment is equality except in type $A_{2 n}^{(2)}$ ). Let $t$ be the collection of indeterminates $t_{\alpha}$ for each root $\alpha$ such that $t_{\alpha}=t_{\alpha^{\prime}}$ if $\alpha$ and $\alpha^{\prime}$ have the same length. Consider the following elements of the group algebra $\mathbb{Q}(q, t) \bar{P}$ :

$$
\Delta:=\left.\prod_{\alpha \in R_{+}^{\text {aff }}} \frac{1-e^{\alpha}}{1-t_{\alpha} e^{\alpha}}\right|_{e^{\delta}=q}, \quad \text { and } \quad \Delta_{1}:=\Delta /\left(\left[e^{0}\right] \Delta\right),
$$

where $\left[e^{0}\right]$ means the coefficient of $e^{0}$ and $R_{+}^{\text {aff }}$ is the set of positive affine real roots. Cherednik's inner product [4] on $\mathbb{Q}(q, t) \bar{P}$ is $\langle f, g\rangle_{q, t}=\left[e^{0}\right]\left(f \bar{g} \Delta_{1}\right)$, where ${ }^{-}$is the involution $\bar{q}=q^{-1}, \bar{t}=t^{-1}, \overline{e^{\lambda}}=e^{-\lambda}$.

The nonsymmetric Macdonald polynomials $E_{\lambda}(q, t) \in \mathbb{Q}(q, t) \bar{P}$ for $\lambda \in \bar{P}$ were introduced by Opdam [48] in the differential setting and Cherednik [4] in general (although here we follow conventions of Haglund, Haiman, Loehr [14, 15]). They are uniquely characterized by two conditions: (Triangularity): $E_{\lambda} \in x^{\lambda}+\mathbb{Q}(q, t)\left\{x^{\mu} \mid \mu<\lambda\right\}$ and (Orthogonality): $\left\langle E_{\lambda}, E_{\mu}\right\rangle_{q, t}=0$ for $\lambda \neq \mu$. Here $<$ is the Bruhat ordering on $\bar{P}$ identified with the set of minimal coset representatives in $\widetilde{W} / \bar{W}$, where $\widetilde{W}$ is the extended affine Weyl group and $\bar{W}$ is the classical Weyl group.

Extending Sanderson's work [53] for type A, Ion [21] showed that for all simply laced untwisted affine root systems, the specialization of the nonsymmetric Macdonald polynomial $E_{\lambda}(q, t)$ at $t=0$ coincides with a specialization of a Demazure character of a level one affine integrable module (see [53, Theorem 6], [21]): Write $t_{\lambda} \in \widetilde{W}$ as $t_{\lambda}=w \tau$, where $w \in W, \tau \in \Sigma$. Then

$$
E_{\lambda}(q, 0)=\left.q^{c} \operatorname{ch}\left(V_{w}\left(\Lambda_{\tau(0)}\right)\right)\right|_{e^{\delta}=q, e^{\Lambda_{0}=1}},
$$

where $c$ is a specific exponent described in [21,53] (and described in Corollary 9.5 below in types $A_{n}^{(1)}$ and $\left.D_{n}^{(1)}\right)$. For $\lambda$ a single row, this relation also follows from combining [37, 20].

Remark 9.4. We have used the conventions of Haglund, Haiman, Loehr [14, 15], which differ from those in [21] by the change of variables $q \rightarrow q^{-1}$.

If $\lambda$ is anti-dominant and $\mathfrak{g}=A_{n}^{(1)}$ or $D_{n}^{(1)}$, we can apply Theorem 6.1 and Corollary 7.6 to give a connection with KR crystals, along with their energy.

Corollary 9.5. Fix $\mathfrak{g}=A_{n}^{(1)}$ or $D_{n}^{(1)}$. Fix an anti-dominant weight $\lambda$, and write $\lambda=$ $-\left(\omega_{r_{1}^{*}}+\cdots+\omega_{r_{N}^{*}}\right)$. Let $B=B^{r_{N}, 1} \otimes \cdots \otimes B^{r_{1}, 1}$. Then

$$
P_{\lambda}(q, 0)=E_{\lambda}(q, 0)=\sum_{b \in B} q^{-D(b)} e^{\mathrm{wt}(b)} .
$$


Proof. The equality $P_{\lambda}(q, 0)=E_{\lambda}(q, 0)$ is [21, Theorem 4.2]. By Theorem 6.1 and Corollary 8.3 (noting that $c_{r}=1$ for types $A_{n}^{(1)}$ and $D_{n}^{(1)}$ as in Figure 4), equation (9.4) implies

$$
P_{\lambda}(q, 0)=q^{c} \sum_{b \in B} q^{-D(b)+D\left(u_{B}\right)} e^{\mathrm{wt}(b)} .
$$

It remains to show that $c=-D\left(u_{B}\right)$. Note that the generator $v=v_{r_{N}, 1} \otimes \cdots \otimes v_{r_{1}, 1} \in B$ has weight $\operatorname{wt}(v)=-\lambda^{*}=w_{0}(\lambda)$. Since $P_{\lambda}(q, 0)$ is symmetric, the coefficient of $e^{w_{0}(\lambda)}$ is the same as the coefficient of $e^{\lambda}$, which by definition of the Macdonald polynomials, is 1 . Hence we obtain the condition $c+D\left(u_{B}\right)-D(v)=0$ which implies by our normalization $D(v)=0$ that $c=-D\left(u_{B}\right)$.

The restriction to types $A_{n}^{(1)}$ and $D_{n}^{(1)}$ in Corollary 9.5 stems from the use of Ion's result [21]. An extension of Corollary 9.5 to type $C_{n}^{(1)}$ follows from [40, 41]. It would be interesting to determine if this result continues to hold in other types.

Remark 9.6. In order to match our notation for nonsymmetric Macdonald polynomials, we index symmetric Macdonald polynomials by anti-dominant weights. Many people (see for example $[24,44]$ ) index Macdonald polynomials by dominant weights. The correct conversion between these conventions is that, for a dominant integral weight $\lambda, P_{\lambda}$ in the above references is denoted by $P_{w_{0}(\lambda)}$ here.

Corollary 9.5 implies that the coefficients in the expansion of the symmetric Macdonald polynomial $P_{\lambda}(q, 0)$ at $t=0$ in terms of the irreducible characters $\operatorname{ch}(V(\mu))$ coincide with $X\left(\mu ; B^{r_{N}, c_{r_{N}}} \otimes \cdots \otimes B^{r_{1}, c_{r_{1}}}\right)$ of (9.2). In formulas

$$
P_{\lambda}(q, 0)=\sum_{\mu} X(\mu ; B) \operatorname{ch}(V(\mu)),
$$

where $B=B^{r_{N}, c_{r_{N}}} \otimes \cdots \otimes B^{r_{1}, c_{r_{1}}}$ with the $r_{i}$ determined from $\lambda$ as in Theorem 6.1.

Remark 9.7. In the case $\mathfrak{g}=A_{n}^{(1)}$, one can define nonsymmetric Macdonald polynomials $E_{\lambda}(q, t)$ for any $\mathfrak{g l}_{n}$ weight $\lambda$ (although if $\lambda$ and $\mu$ correspond to the same $\mathfrak{s l}_{n}$ weight, these only differ by a scalar). Letting $\bar{P}^{\prime}$ denote the lattice of $\mathfrak{g l}_{n}$ weights, there is a natural injection

$$
\begin{aligned}
\mathbb{Q}(q, t) \bar{P}^{\prime} & \rightarrow \mathbb{Q}(q, t)\left[x_{1}^{ \pm 1}, \ldots, x_{n}^{ \pm 1}\right] \\
e^{\lambda} & \mapsto x_{1}^{\lambda_{1}} x_{2}^{\lambda_{2}} \cdots x_{n}^{\lambda_{n}},
\end{aligned}
$$

so we can identify $E_{\lambda}(q, t)$ with an actual polynomial, and we do so in the examples below by writing $E_{\lambda}(x ; q, t)$.

Example 9.8. The Macdonald polynomial of type $A_{2}^{(1)}$ indexed by the anti-dominant weight $(0,0,2)$ is given by

$$
P_{(0,0,2)}(x ; q, 0)=x_{1}^{2}+(q+1) x_{1} x_{2}+x_{2}^{2}+(q+1) x_{1} x_{3}+(q+1) x_{2} x_{3}+x_{3}^{2} .
$$


By Corollary 9.5, this is given by the character of $B^{2^{*}, 1} \otimes B^{2^{*}, 1}$, where the power of $q$ counts $E^{\text {int }}$. Here $2^{*}=1$, so we consider the KR crystal $B^{1,1} \otimes B^{1,1}$. Under the map from Theorem 6.1, this is isomorphic as a classical crystal to $B_{s_{2} s_{1} s_{0} s_{2}}\left(\Lambda_{2}\right)$, and there are enough 0 arrows in this Demazure crystal to calculate $E^{\text {int }}$. The KR crystal is then given as follows, where to make the picture cleaner we have only included arrows which survive in the Demazure crystal. Note that the ground state path is $2 \otimes 1$.

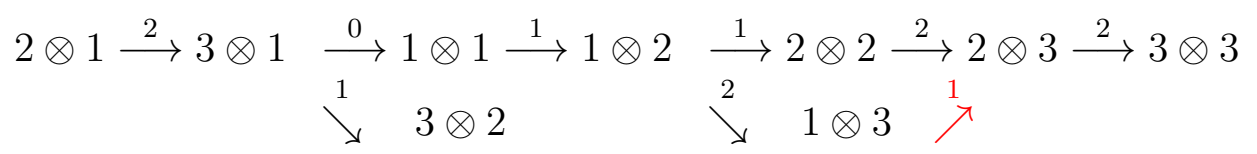

The black arrows are all arrows that appear in $f_{2}^{n_{1}} f_{1}^{n_{2}} f_{0}^{n_{3}} f_{2}^{n_{4}}(2 \otimes 1)$ for some exponents $n_{i} \geqslant 0$, which give all vertices of the Demazure crystal, but not all arrows. The red arrows are the additional arrows in the Demazure crystal. We calculate that $D(2 \otimes 1)=$ $D(3 \otimes 1)=D(3 \otimes 2)=-1$, and the rest of the elements of $B^{1,1} \otimes B^{1,1}$ have $D=0$. This confirms Corollary 9.5 in this case.

The connection with $\mathrm{KR}$ crystals can also be used to compute all nonsymmetric Macdonald polynomials specialized at $t=0$. Fix $\lambda$ which need not be anti-dominant, and define $w, \tau$ as in (9.4). There is a unique minimal $w^{\prime} \in W_{0}$ such that $w^{\prime}(\lambda)$ is an anti-dominant weight. Then $w^{\prime} w\left(\left(w^{\prime}\right)^{-1}\right)^{\tau} \tau$ is a translation by an anti-dominant weight, where the superscript $\tau$ means conjugation by $\tau$. Also, $B_{w}\left(\Lambda_{\tau(0)}\right)$ embeds in $B_{w^{\prime} w\left(\left(w^{\prime}\right)^{-1}\right)^{\tau}}\left(\Lambda_{\tau(0)}\right)=B_{w^{\prime} w}\left(\Lambda_{\tau(0)}\right)$, where the final equality follows because $w^{\prime} \in W_{0}$. Let $j: B_{w^{\prime} w}\left(\Lambda_{\tau(0)}\right) \rightarrow B$ be the isomorphism from Theorem 6.1, where $B$ is the appropriate composite KR crystal, and let $B^{\prime}=j\left(B_{w}\left(\Lambda_{\tau(0)}\right)\right)$. Then in types $A_{n}^{(1)}$ and $D_{n}^{(1)}$ we have

$$
E_{\lambda}(q, 0)=\sum_{b \in B^{\prime}} q^{-D(b)} e^{\mathrm{wt}(b)}
$$

where $D$ is calculated in the ambient composite KR crystal $B$. The subset $B^{\prime}$ of $B$ can be described as $\left\{f_{i_{k}}^{n_{k}} \cdots f_{i_{1}}^{n_{1}} u_{B} \mid n_{1}, \ldots, n_{k} \geqslant 0\right\}$, where $s_{i_{k}} \cdots s_{i_{1}}$ is any reduced expression for $w$.

Example 9.9. Again consider type $A_{2}^{(1)}$, and take the nonsymmetric Macdonald polynomial

$$
E_{(0,2,0)}(x ; q, 0)=x_{1}^{2}+(q+1) x_{1} x_{2}+x_{2}^{2}+q x_{1} x_{3}+q x_{2} x_{3} .
$$

Then $w, \tau$ from (9.4) are $w=s_{1} s_{0} s_{2} s_{1}$ and $\tau=0 \rightarrow 2 \rightarrow 1 \rightarrow 0$. The shortest $w^{\prime} \in W_{0}$ so that $w^{\prime}(0,2,0)$ is anti-dominant is $w^{\prime}=s_{2}$. Thus the above argument along with Example 9.8 shows that $B_{w}\left(\Lambda_{\tau(0)}\right)=B_{s_{1} s_{0} s_{2} s_{1}}\left(\Lambda_{2}\right)=B_{s_{1} s_{0} s_{2}}\left(\Lambda_{2}\right)$ embeds into $B=B^{1,1} \otimes$ $B^{1,1}$ (as a classical crystal), and a simple verification shows that the image $B^{\prime}$ is everything but $1 \otimes 3,2 \otimes 3$ and $3 \otimes 3$. This indeed verifies that $E_{(0,2,0)}(x ; q, 0)$ is given by $(9.6)$.

Example 9.10. Consider the Macdonald polynomial of type $A_{2}^{(1)}$

$$
P_{(0,1,2)}(x ; q, 0)=x_{1}^{2} x_{2}+x_{1} x_{2}^{2}+x_{1}^{2} x_{3}+(q+2) x_{1} x_{2} x_{3}+x_{2}^{2} x_{3}+x_{1} x_{3}^{2}+x_{2} x_{3}^{2} .
$$


Our results say that this is given by the character of the affine crystal $B$, where $B=$ $B^{2,1} \otimes B^{1,1}$. The ground state path is $\begin{aligned} & 3 \\ & 2\end{aligned} \otimes 1$. As a classical crystal, $B$ consists of two components, with highest weight elements $\begin{aligned} & 3 \\ & 2\end{aligned} \otimes 1$ and $\begin{aligned} & 2 \\ & 1\end{aligned} \otimes 1$ of weight 0 and $\omega_{1}+\omega_{2}$ respectively. One can check that $D\left(\begin{array}{l}3 \\ 2\end{array} \otimes 1\right)=-1$, and $D\left(\begin{array}{l}2 \\ 1\end{array} \otimes 1\right)=0$. This indeed confirms

$$
P_{(0,1,2)}(x, q, 0)=q+\operatorname{ch}\left(V\left(\omega_{1}+\omega_{2}\right)\right) .
$$

\section{3 q-deformed Whittaker functions}

Gerasimov, Lebedev, Oblezin [13, Theorem 3.2] showed that $q$-deformed $\mathfrak{g l}_{n}$-Whittaker functions are Macdonald polynomials specialized at $t=0$. As above this also gives a link to Demazure characters, and hence by the results in Section 7 to KR crystals graded by their energy functions. It would be interesting to generalize this to other types (in particular type $D_{n}^{(1)}$, where both Ion's results [21] and our results from Section 7 hold). The $q$-deformed $\mathfrak{g l}_{n}$-Whittaker functions are simultaneous eigenfunctions of a $q$ deformed Toda chain, which might serve as a starting point for this generalization. See also the recent paper by Braverman and Finkelberg [1]. Brubaker, Bump and Licata [2] constructed a natural basis of the Iwahori fixed vectors in the Whittaker model using Demazure-Lusztig operators. This gives another avenue to the link between Demazure characters and Whittaker functions.

\section{Acknowledgments}

We would like to thank Dan Bump, Ghislain Fourier, Stavros Kousidis, Cristian Lenart, Sergey Oblezin, and Masato Okado for enlightening discussions, and Nicolas Thiéry for his help with Sage. Most of the KR crystals have been implemented in the open-source mathematics system Sage (sagemath.org) by the first author. A thematic tutorial on crystals and affine crystals written by Dan Bump and the first author is available in Sage. A copy can be found at

$$
\begin{aligned}
& \text { http://www. math. ucdavis.edu/ }{ }^{\sim} \text { anne/sage/lie/crystals.html } \\
& \text { http://www.math. ucdavis.edu/ }{ }^{\sim} \text { anne/sage/lie/affine_crystals.html. }
\end{aligned}
$$

We would like to thank the Fields Institute in Toronto and the Hausdorff Institut in Bonn for their hospitality, where part of this work was done. AS was in part supported by NSF grants DMS-0652641, DMS-0652652, and DMS-1001256. PT was partially supported by NSF grant DMS-0902649. 


\section{References}

[1] A. Braverman, M. Finkelberg, Weyl modules and q-Whittaker functions, preprint arXiv: 1203.1583.

[2] B. Brubaker, D. Bump, A. Licata, Whittaker functions and Demazure operators, arXiv:1111.4230.

[3] V. Chari, On the fermionic formula and the Kirillov-Reshetikhin conjecture, Internat. Math. Res. Notices 12 (2001), 629-654.

[4] I. Cherednik, Nonsymmetric Macdonald polynomials, Internat. Math. Res. Notices 10 (1995), 483-515.

[5] V. Chari, A. Pressley, Quantum affine algebras and their representations, CMS Conf. Proc., 16, Amer. Math. Soc., Providence, RI, 1995, 59-78.

[6] V. Chari, A. Pressley, Twisted quantum affine algebras, Comm. Math. Phys. 196 (1998), no. 2, 461-476. arXiv:q-alg/9611002

[7] M. Demazure, Désingularisation des variétés de Schubert gén'eralisées, Ann. Sci. École Norm. Sup. (4) 7 (1974), 53-88.

[8] G. Fourier, P. Littelmann, Tensor product structure of affine Demazure modules and limit constructions, Nagoya Math. J. 182 (2006), 171-198. arXiv:math/0412432

[9] G. Fourier, P. Littelmann, Weyl modules, Demazure modules, KR-modules, crystals, fusion products and limit constructions, Adv. Math. 211 (2007), no. 2, 566-593. arXiv:math/0509276

[10] G. Fourier, M. Okado, A. Schilling Kirillov-Reshetikhin crystals of nonexceptional type, Advances in Math. 222 (2009) 1080-1116 arXiv:0810.5067

[11] G. Fourier, M. Okado, A. Schilling, Perfectness of Kirillov-Reshetikhin crystals for nonexceptional type, Contemp. Math. 506 (2010) 127-143. arXiv:0811.1604

[12] G. Fourier, A. Schilling, M. Shimozono, Demazure structure inside KirillovReshetikhin crystals, J. Algebra 309 (2007) 386-404. arXiv:math/0605451

[13] A. Gerasimov, D. Lebedev, S. Oblezin, On q-deformed $g l(l+1)$-Whittaker function III, Lett. Math. Phys. 97 (2011), no. 1, -24. arXiv:0805.3754

[14] M. Haiman, Cherednik algebras, Macdonald polynomials and combinatorics, International Congress of Mathematicians. Vol. III, 843-872, Eur. Math. Soc., Zürich, 2006.

[15] J. Haglund, M. Haiman, N. Loehr, A combinatorial formula for nonsymmetric Macdonald polynomials, Amer. J. Math. 130 (2008), no. 2, 359-383. arXiv:math/0601693

[16] G. Hatayama, A. Kuniba, M. Okado, T. Takagi, Z. Tsuboi, Paths, crystals and fermionic formulae, MathPhys Odyssey 2001, 205-272, Prog. Math. Phys. 23, Birkhäuser Boston, Boston, MA, 2002. arXiv:math/0102113

[17] G. Hatayama, A. Kuniba, M. Okado, T. Takagi, Y. Yamada, Remarks on fermionic formula Recent developments in quantum affine algebras and related topics (Raleigh, 
NC, 1998), 243-291, Contemp. Math., 248, Amer. Math. Soc., Providence, RI, 1999. arXiv:math/9812022v3

[18] J. Hong, S.-J. Kang, Introduction to quantum groups and crystal bases, Graduate Studies in Mathematics, 42, American Mathematical Society, Providence, RI, 2002. xviii+307 pp.

[19] D. Hernandez, Kirillov-Reshetikhin conjecture: the general case, Int. Math. Res. Not. IMRN 2010, no. 1, 149-193.

[20] K. Hikami, Representation of the Yangian invariant motif and the Macdonald polynomial, J. Phys. A 30 (1997), no. 7, 2447-2456.

[21] B. Ion. Nonsymmetric Macdonald polynomials and Demazure characters, Duke Math. J. 116(2) (2003), 299-318. arXiv:math/0105061

[22] B. Jones, A. Schilling Affine structures and a tableau model for $E_{6}$ crystals, J. Algebra 324 (2010) 2512-2542. arXiv:0909. 2442

[23] V. G. Kac, Infinite-dimensional Lie algebras, Third edition, Cambridge University Press, Cambridge, 1990.

[24] F. Knop, Integrality of two variable Kostka functions, J. Reine Angew. Math. 482 (1997), 177-189. arXiv:q-alg/9603027

[25] S-J. Kang, M. Kashiwara, K. C. Misra, T. Miwa, T. Nakashima, A. Nakayashiki, Affine crystals and vertex models, Int. J. Mod. Phys. A 7 (suppl. 1A) (1992), 449484.

[26] S.-J. Kang, M. Kashiwara, K. C. Misra, T. Miwa, T. Nakashima, A. Nakayashiki, Perfect crystals of quantum affine Lie algebras, Duke Math. J. 68 (1992) 499-607.

[27] M. Kashiwara, The crystal base and Littelmann's refined Demazure character formula, Duke Math. J. 71 (1993), no. 3, 839-858.

[28] M. Kashiwara, On crystal bases of the q-analogue of universal enveloping algebras, Duke Math. J. 73 (1994), 383-413.

[29] M. Kashiwara, Similarity of crystal bases, Lie algebras and their representations (Seoul, 1995), 177-186, Contemp. Math., 194, Amer. Math. Soc., Providence, RI, 1996.

[30] M. Kashiwara, On level-zero representations of quantized affine algebras, Duke Math. J. 112 (2002), no. 1, 117-175. arXiv:math/0010293

[31] M. Kashiwara Level zero fundamental representations over quantized affine algebras and Demazure modules, Publ. Res. Inst. Math. Sci. 41 (2005), no. 1, 223-250. arXiv:math/0309142

[32] M. Kashiwara, K.C. Misra, M. Okado, D. Yamada Perfect crystals for $U_{q}\left(D_{4}^{(3)}\right)$, J. Algebra 317 (2007), no. 1, 392-423. arXiv:math/0610873

[33] M. Kashiwara, T. Nakashima, Crystal graphs for representations of the q-analogue of classical Lie algebras, J. Algebra 165 (1994), no. 2, 295-345. 
[34] A.N. Kirillov, N.Yu. Reshetikhin Representations of Yangians and multiplicities of the inclusions of the irreducible components of the tensor product of representations of simple Lie algebras. Zap. Nauchn. Sem. Leningrad. Otdel. Mat. Inst. Steklov. (LOMI) 160 (1987), Anal. Teor. Chisel i Teor. Funktsii. 8, 211-221, 301; translation in J. Soviet Math. 52 (1990), no. 3, 3156-3164

[35] S. Kumar, Demazure character formula in arbitrary Kac-Moody setting, Invent. Math. 89 (1987), no. 2, 395-423.

[36] A. Kuniba, K. C. Misra, M. Okado, J. Uchiyama, Demazure modules and perfect crystals, Comm. Math. Phys. 192 (1998), no. 3, 555-567. arXiv:q-alg/9607011

[37] A. Kuniba, K. C. Misra, M. Okado, T. Takagi, J. Uchiyama, Characters of Demazure modules and solvable lattice models, Nuclear Phys. B 510 (1998), no. 3, 555-576. arXiv:q-alg/9707004

[38] A. Kuniba, K. C. Misra, M. Okado, T. Takagi, J. Uchiyama, Crystals for Demazure modules of classical affine Lie algebras, J. Algebra 208 (1998), no. 1, 185-215. arXiv: q-alg/9707014

[39] C. Lecouvey, M. Okado, M. Shimozono, Affine crystals, one dimensional sums and parabolic Lusztig q-analogues, Math. Zeitschrift, to appear, DOI: 10.1007/s00209011-0892-9. arXiv: 1002.3715v1.

[40] C. Lenart, From Macdonald polynomials to a charge statistic beyond type A, J. Combin. Theory Ser. A,119 (2012), 683-712. arXiv:1106.3296.

[41] C. Lenart, A. Schilling, Crystal energy functions via the charge in types $A$ and $C$, Math. Zeitschrift, to appear. arXiv:1107.4169.

[42] P. Littelmann, Paths and root operators in representation theory, Ann. of Math. (2) 142 (1995), no. 3, 499-525.

[43] P. Littelmann, Crystal graphs and Young tableaux, J. Algebra 175 (1995), no. 1, $65-87$.

[44] D. Marshall, Symmetric and nonsymmetric Macdonald polynomials. On combinatorics and statistical mechanics, Ann. Comb. 3 (1999), no. 2-4, 385-415. arXiv: math $/ 9812080$

[45] O. Mathieu, Formules de Demazure-Weyl, et généralisation du théorème de BorelWeil-Bott, C. R. Acad. Sci. Paris Sér. I Math. 303 (1986), no. 9, 391-394.

[46] K. Naoi, Weyl modules, Demazure modules and finite crystals for non-simply laced type, preprint arXiv: 1012.5480

[47] K. Naoi, Demazure crystals and tensor products of perfect Kirillov-Reshetikhin crystals with various levels, preprint arXiv:1108.3139

[48] E. Opdam, Harmonic analysis for certain representations of graded Hecke algebras, Acta Math. 175 (1995), no. 1, 75-121.

[49] M. Okado Existence of crystal bases for Kirillov-Reshetikhin modules of type D, Publ. Res. Inst. Math. Sci. 43 (2007), no. 4, 977-1004. arXiv:math.QA/0610874 
[50] M. Okado, A. Schilling, Existence of Kirillov-Reshetikhin crystals for nonexceptional types Represent. Theory 12 (2008) 186-207. Erratum: Represent. Theory 12 (2008), 499-500. arXiv:0706.2224

[51] M. Okado, A. Schilling, M. Shimozono, Virtual crystals and fermionic formulas of type $D_{n+1}^{(2)}, A_{2 n}^{(2)}$, and $C_{n}^{(1)}$, Representation Theory 7 (2003) 101-163. arXiv:math/0105017

[52] M. Okado, A. Schilling, M. Shimozono, Virtual crystals and Kleber's algorithm, Commun. Math. Phys. 238 (2003) 187-209. arXiv:math/0209082

[53] Y. Sanderson, On the connection between Macdonald polynomials and Demazure characters, J. Algebraic Combin. 11 (2000) 269-275.

[54] M. Shimozono, Affine type A crystal structure on tensor products of rectangles, Demazure characters, and nilpotent varieties, J. Algebraic Combin. 15 (2002), no. 2, $151-187$.

[55] A. Schilling, Combinatorial structure of Kirillov-Reshetikhin crystals of type $D_{n}^{(1)}$, $B_{n}^{(1)}, A_{2 n-1}^{(2)}$, J. Algebra 319 (2008) 2938-2962. arXiv:0704. 2046

[56] Y. Yamane Perfect crystals of $U_{q}\left(G_{2}^{(1)}\right)$. J. Algebra 210 (1998), no. 2, 440-486. arXiv:q-alg/9712012 